\title{
Optimal Population Codes for Space: Grid Cells Outperform Place Cells
}

\author{
Alexander Mathis \\ mathis@bio.lmu.de \\ Andreas V. M. Herz \\ herz@bio.lmu.de \\ Martin Stemmler \\ stemmler@bio.lmu.de \\ Bernstein Center for Computational Neuroscience Munich; Graduate School \\ of Systemic Neuroscience and Division of Neurobiology, \\ Ludwig-Maximilians-Universität München, 82152 Martinsried, Germany
}

Rodents use two distinct neuronal coordinate systems to estimate their position: place fields in the hippocampus and grid fields in the entorhinal cortex. Whereas place cells spike at only one particular spatial location, grid cells fire at multiple sites that correspond to the points of an imaginary hexagonal lattice. We study how to best construct place and grid codes, taking the probabilistic nature of neural spiking into account. Which spatial encoding properties of individual neurons confer the highest resolution when decoding the animal's position from the neuronal population response? A priori, estimating a spatial position from a grid code could be ambiguous, as regular periodic lattices possess translational symmetry. The solution to this problem requires lattices for grid cells with different spacings; the spatial resolution crucially depends on choosing the right ratios of these spacings across the population. We compute the expected error in estimating the position in both the asymptotic limit, using Fisher information, and for low spike counts, using maximum likelihood estimation. Achieving high spatial resolution and covering a large range of space in a grid code leads to a trade-off: the best grid code for spatial resolution is built of nested modules with different spatial periods, one inside the other, whereas maximizing the spatial range requires distinct spatial periods that are pairwisely incommensurate. Optimizing the spatial resolution predicts two grid cell properties that have been experimentally observed. First, short lattice spacings should outnumber long lattice spacings. Second, the grid code should be self-similar across different lattice spacings, so that the grid field always covers a fixed fraction of the lattice period. If these conditions are satisfied and the spatial "tuning curves" for each neuron span the same range of firing rates, then the resolution of the grid code easily exceeds that of the best possible place code with the same number of neurons. 


\section{Introduction}

An animal's position and heading in world coordinates is reflected in coordinated neural firing patterns within different subnetworks of the brain, most notably the hippocampus, subiculum, and entorhinal cortex (O'Keefe \& Dostrovsky, 1971; O’Keefe, 1976; Taube, Muller, \& Ranck, 1990a, 1990b; Fyhn, Molden, Witter, Moser, \& Moser, 2004; Hafting, Fyhn, Molden, Moser, \& Moser, 2005; Boccara et al., 2010). In rodents, these subnetworks have evolved at least two distinct representations for encoding spatial location: in the hippocampus proper, place cells fire only at a single, specific location in space, whereas in the medial entorhinal cortex (mEC), grid cells build a hexagonal lattice representation of physical space, such that each cell fires whenever the animal moves through a firing field centered at a cell-specific lattice point.

How accurately can an animal determine its location using one of these two distinct encoding schemes for space? Most neurons in cortex spike irregularly and unreliably (Softky \& Koch, 1993; Shadlen \& Newsome, 1998), and cells in the hippocampal-entorhinal loop are no exception (Fenton \& Muller, 1998; Kluger, Mathis, Stemmler, \& Herz, 2010). As the animal moves through space, it spends only a brief moment in each firing field of a grid cell or the firing field of a place cell, eliciting no more than a handful of unreliable spikes. Grid cells, for instance, often spike only once or twice during a single pass through a firing field (Reifenstein, Stemmler, \& Herz, 2010). Hence, for both codes, precise information about position can be gained only from a population of grid and place cells, respectively. If all grid cells share the same lattice length scale, the same pattern of spikes across the population corresponds to different locations in space, leading to catastrophic errors in estimating position. How different lattices can be combined to resolve the ambiguity introduced by the multiplicity of firing fields is crucial for navigation and might explain the variation of the spatial periods along the dorso-ventral axis for the mEC (Brun et al., 2008).

The goal of this letter is to answer the question of how grid codes should be constructed and relate these to the resolution of population codes. Singlepeaked place fields are analogous to the tuning curves for orientation in visual and motor cortices, for which the questions of neuronal coding and optimal tuning widths have been investigated extensively (Paradiso, 1988; Seung \& Sompolinsky, 1993; Brunel \& Nadal, 1998; Zhang \& Sejnowski, 1999; Pouget, Deneve, Ducom, \& Latham, 1999; Bethge, Rotermund, \& Pawelzik, 2002; Brown \& Bäcker, 2006; Bobrowski, Meir, \& Eldar, 2009). Theoretical studies on the coding properties of grid cells (Burak, Brookings, \& Fiete, 2006; Fiete, Burak, \& Brookings, 2008) have dealt with the spatial range encoded by populations of grid cells, without assuming an explicit noise model. Here, our focus will be on neither the spatial range nor how gridlike firing patterns arise (Fuhs \& Touretzky, 2006; McNaughton, Battaglia, Jensen, Moser, \& Moser, 2006; Burgess, Barry, \& O’Keefe, 2007; 
Kropff \& Treves, 2008; Burak \& Fiete, 2009; Remme, Lengyel, \& Gutkin, 2010; Zilli \& Hasselmo, 2010; Mhatre, Gorchetchnikov, \& Grossberg, 2010), nor how grid fields can lead to place fields (Fuhs \& Touretzky, 2006; Solstad, Moser, \& Einevoll, 2006; Rolls, Stringer, \& Elliot, 2006; Franzius, Vollgraf, \& Wiskott, 2007; Si \& Treves, 2009; Cheng \& Loren, 2010). Rather, we extract general observations about grid and place cells from experimental findings and relate these to the resolution of population codes. In addition to comparing grid and place codes quantitatively, we derive optimal parameter regimes for both codes. Using the hypothesis that neuronal populations code efficiently (Attneave, 1954; Barlow, 1959), we can then make predictions about grid cell properties in the $\mathrm{mEC}$.

The comparison will be carried out in the framework of Poisson rate coding for the position of an animal along a one-dimensional path, typically a linear track (Hafting, Fyhn, Bonnevie, Moser, \& Moser, 2008; Brun et al., 2008). A place cell is characterized by a single firing field with a given spatial center and width; for grid cells, one measures the spatial period and phase of the regularly spaced lattice of firing fields. These parameters define families of tuning curves for population models of spatial coding. Based on maximum likelihood decoding, we estimate the distortion, or average error, in recovering the animal's position. Asymptotically, given enough neurons and a long enough time to observe the firing rate, the distortion becomes analytically calculable. The Cramér-Rao bound states that the inverse of the Fisher information yields the minimum achievable square error, provided the estimator is unbiased; furthermore, maximum likelihood decoding attains this bound (Lehmann \& Casella, 1998). In the context of neural population coding, many authors have calculated the Fisher information (Paradiso, 1988; Seung \& Sompolinsky, 1993; Brunel \& Nadal, 1998; Zhang \& Sejnowski, 1999; Pouget et al., 1999; Eurich \& Wilke, 2000; Wilke \& Eurich, 2002; Bethge et al., 2002; Brown \& Bäcker, 2006). However, it is also known that no such estimator will attain the lower bound if the neurons have Poisson spike statistics and the expected number of spikes is low, even when a neuron is firing at its maximal rate (Bethge et al., 2002). In other words, if the product of the firing rate $f_{\max }$ and the time window $T$ for counting spikes obeys $f_{\max } T \approx 1$, the Fisher information greatly exaggerates the true spatial resolution of the population code. If one takes the time window for readout to be one cycle of the ongoing $7 \mathrm{~Hz}$ to $12 \mathrm{~Hz}$ theta rhythm during movement, the natural timescale for grid and place cells is short compared to the typical firing rates in these cells. Under these conditions, the asymptotic error and the true error can diverge, so that the parameters for an optimal grid code can be found only numerically. Maximum likelihood decoding is computationally expensive, so we treat the case of populations encoding a one-dimensional stimulus in detail. Multiple stimulus dimensions correspond to a product space in the mathematical sense; under ideal conditions, the errors across stimulus dimensions add. Hence, studying the one-dimensional case will be 


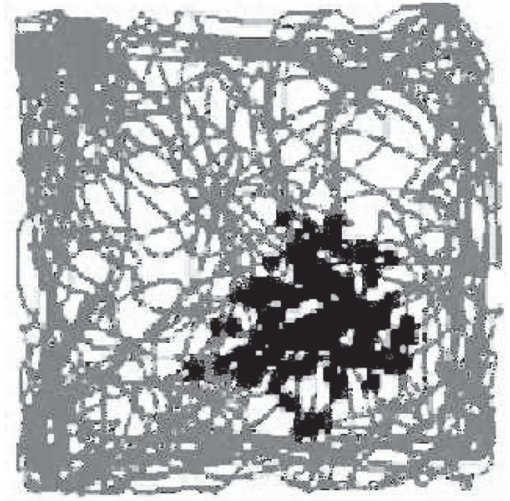

(a)

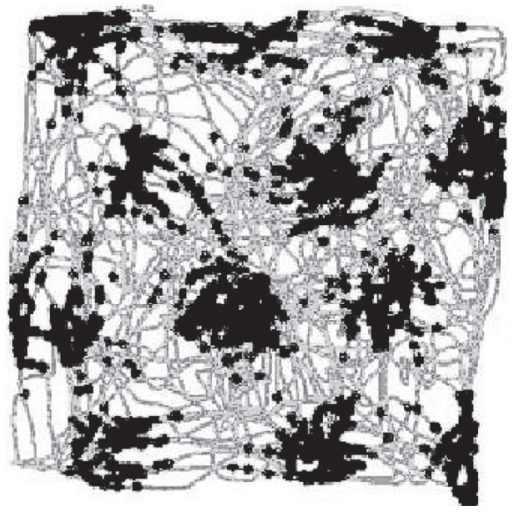

(b)

Figure 1: Firing patterns for a place and grid cell. (a) A place cell spikes only when the animal is within a single region of space called the place field. Gray lines depict the trajectory of a rat in a square arena. The superimposed black dots mark the rat's location when this CA1 cell in hippocampus fired a spike. (Figure adapted from Jeffery, 2008, with permission.) (b) In contrast, a grid cell from entorhinal cortex fires at multiple spatial locations, which form a hexagonal lattice. Three neighboring firing fields span a nearly equilateral triangle. (Figure adapted from Hafting et al., 2005, with permission.)

illustrative for how general grid codes should be constructed, as we will discuss.

Some of the results here have been presented in a briefer format in Mathis, Stemmler, and Herz (2010).

\section{Grid Code Schemes}

The place code is a classical instance of a population code (Wilson \& McNaughton, 1993), wherein each position in space is represented by the activity of a large number of place cells (see Figure 1a) with intersecting place fields. The set of well-localized place fields forms a dense cover of the explored space, so that the set of simultaneously active place cells yields an accurate estimate of the animal's position. Additional precision in estimating the position can be gained from the spatial profile of how individual place cells map position into a firing rate-the place cell's "tuning curve" (Paradiso, 1988; Seung \& Sompolinsky, 1993; Zhang \& Sejnowski, 1999). Early models considered cells with single fields and a standard tuning curve for each cell. Yet the width of the place fields grows along the dorsoventral axis (Kjelstrup et al., 2008), and ventral CA3 cells are more likely to 
have more than one place field (Leutgeb, Leutgeb, Moser, \& Moser, 2007; Fenton et al., 2008). As we will show, both of these properties can improve the resolution, but only marginally.

A grid code, in contrast, is harder to read out. The firing of a single grid cell (see Figure 1b) implies that the animal could be at any one of a range of different locations, without specifying which one. A clear-cut estimate of position becomes possible by taking into account the properties of neighboring grid cells, each characterized by a regular lattice of locations at which the cell fires. For neighboring grid cells, the lattices share similar spatial periods and orientations but are spatially translated (Hafting et al., 2005; Sargolini et al., 2006; Doeller, Barry, \& Burgess, 2010). A single grid cell thus signals the spatial phase of the animal's location relative to the lattice. Taking a subset from the local grid cell population that spans all phases is tantamount to discretizing the spatial phase and forms the basis for defining a grid module: an ensemble of grid cells that share the same lattice properties but have different spatial phases. Along the dorsolateral axis of the $\mathrm{mEC}$, the typical spatial period grows from values of around 20 centimeters up to several meters (Fyhn et al., 2004; Giocomo, Zilli, Fransén, \& Hasselmo, 2007; Brun et al., 2008), while the ratio of grid field width to spatial period remains constant (Hafting et al., 2005; Brun et al., 2008).

The range and precision of the grid code's representation of space crucially depend on how the spatial periods of different modules are arranged. In the most extreme case, the combination of spatial periods could yield a population code with a high resolution but a short range, or vice versa. Many grid codes will have mixed properties, implying no hard trade-off between range and precision. Let us, nevertheless, first compare two extremes of grid coding. In the first, the spatial periods themselves span a wide range, effectively subdividing space; in the second, the spatial periods are similar yet incommensurate, so that the phases represented in the population response are unique for each position across a wide range of space. We call the first scheme the nested interval scheme, illustrated in Figure 2a. Imagine that the spatial periods $\lambda_{i}$ are ordered, $\lambda_{1}>\lambda_{2}>\cdots>\lambda_{L}$. For each $\lambda_{i}$, assume that there are $M$ grid cells that share this spatial period but have lattices that are shifted relative to each other. The $M$ cells will represent the equidistant phases $2 \pi j / M$ with $j \in\{0,1, \ldots, M-1\}$. Such a grid encodes positions smaller than $\lambda_{1}$ precisely and effectively in a step-bystep fashion. Module 1 provides only coarse information about the position estimate, with a resolution of $\lambda_{1} / M$. Module 2 , although itself ambiguous within the range $\left[0, \lambda_{1}\right]$, adds resolution within each of the $M$ subintervals of length $\lambda_{1} / M$. Likewise, module 3 adds further precision, and so forth. An analog clock works the same way: within a 12 hour span, the minute and second hand are ambiguous per se. While the hour hand could, in principle, encode the time of the day down to microsecond precision, there is a limit to the angular resolution of the human eye, whereas the combination of all hands is easy to read. Similarly, the nested interval scheme can resolve 


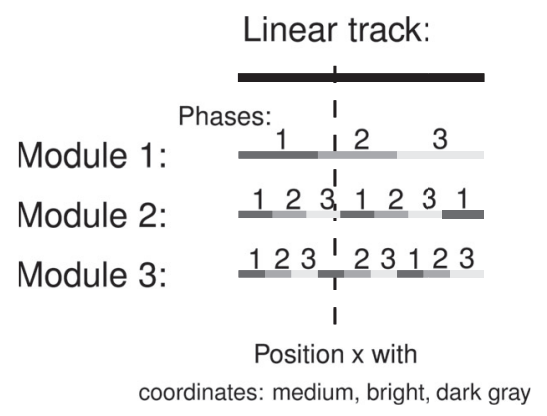

(a)

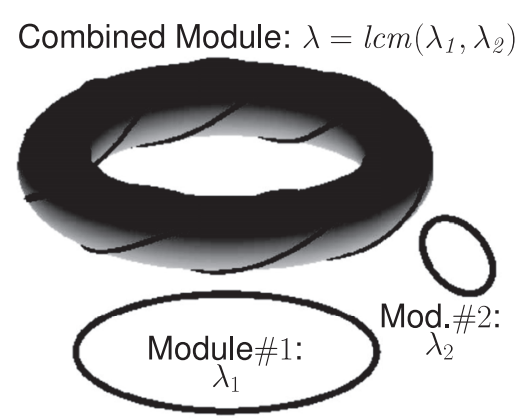

(b)

Figure 2: (a) Nested interval scheme. Example with three clearly different spatial periods and three discrete phases each. The first module gives coarse spatial information that is further refined by the other two modules. By themselves, the other modules provide ambiguous spatial information on the range; together, they effectively subdivide the unit interval. (b) Modular arithmetic scheme. The two periodic variables depicted by the circles with different spatial periods $\lambda_{1}$ and $\lambda_{2}$ can lead to an elongation of the coding range. Geometrically this can be seen by considering a particle wandering with the same increment in each variable on the Cartesian product of the two circles, which is a torus. The trajectory of this particle will close after length $\operatorname{lcm}\left(\lambda_{1}, \lambda_{2}\right)$, the least common multiple, as described in the text.

the position with high accuracy, even though the individual modules lack either spatial precision or spatial range. Unlike the clock, the periods $\lambda_{i}$ are not necessarily integer multiples of each other, that is, $\lambda_{i} \nmid \lambda_{i-1}$. In this case, the range, which is the longest distance that is unambiguously coded by the modules, can be much larger than the largest spatial period $\lambda_{1}$. Extending the range beyond the largest spatial period is the key idea behind the modular arithmetic scheme (Fiete et al., 2008), which is the alternative to nested interval coding.

Consider two one-dimensional modules with spatial periods 12 and 17. One can represent each module as a circle $S^{1}$, whose circumference matches the period. Geometrically, spatial positions are mapped onto the product of these two circles, which is a torus $T^{2}=S^{1} \times S^{1}$. The mapping of spatial position is unique up to the point at which

$$
\begin{aligned}
\Pi:[0, \infty) & \rightarrow T^{2} \\
x & \mapsto(\bmod (x, 12), \quad \bmod (x, 17))
\end{aligned}
$$

closes in on itself for the first time (i.e., $\min _{x>0} \Pi(x)=\Pi(0)$ ). As the integers 12 and 17 have no common divisor, the period is $204=12 \cdot 17$, the least 
common multiple of the two spatial periods. ${ }^{1}$ This principle is illustrated in Figure $2 b$. By induction, one can show that the range of a sequence of spatial periods $\left\{\lambda_{1}, \lambda_{2}, \ldots \lambda_{L}\right\}$ is given by the least common multiple of this sequence $\operatorname{lcm}\left(\lambda_{1}, \lambda_{2}, \ldots \lambda_{L}\right)$.

At best, an ideal, noiseless grid code with integer periods has a range that is the product of the spatial length scales (Fiete et al., 2008). A small change in the periods, however, can lead to a dramatic reduction in the range. For instance, changing the periods from 12 and 17 to 12 and 18 reduces the range from 204 to merely 36, the least common multiple of 12 and 18. In general, for two positive real numbers representing the spatial periods, the combined period is given by

$$
\operatorname{lcm}(x, y)= \begin{cases}\infty & x / y \notin \mathbb{Q} \\ n \cdot x & x / y \in \mathbb{Q} \text { with } x=\frac{m}{n} \cdot y \\ & \text { for } m, n \in \mathbb{N}, \text { without common divisor. }\end{cases}
$$

This function is highly discontinuous. For every pair of periods $\left(\lambda_{1}, \lambda_{2}\right) \in \mathbb{Q}$, one can find an arbitrarily close pair of rational spatial periods with an arbitrarily large $l \mathrm{~cm}$. In contrast, within any vicinity $\left(\lambda_{1}, \lambda_{2}\right)$, a smallest least common multiple exists.

An even more severe problem than the sensitivity of the range lurks. For the spatial periods from the example above, $\lambda_{1}=12$ and $\lambda_{2}=17$, changing the modular coordinates from $(0,0)$ to $(1,0)$ implies a jump in position from 0 to 85 , which is almost half of the range. Small errors in the phase can thus lead to huge mistakes in the position estimate. Choosing more closely spaced periods limits the magnitude of such an error, yet a unit step in any one coordinate represents a shift in the position by at least one spatial period.

In principle, the grid lattice need not be regular, nor need a grid cell share the same lattice spacing with other grid cells. We will not consider the most general case here but make the prior assumption of both periodicity and modularity, two features that could facilitate the downstream readout of the neuronal population's response. We will construct nested interval and modular arithmetic codes by sampling from the space of different possible spatial periods in these ways:

- Deterministic ensembles. Given $N$ cells, assign an equal number of cells to a set of modules whose spatial periods are defined as follows: starting with an initial module with spatial period $\lambda=1$, let each

\footnotetext{
${ }^{1}$ In contrast to the watch example, the two periods should not have a common divisor. Since a second divides a minute and a minute divides an hour, a standard analog watch does not represent more than the maximal 12 hour period.
} 
successive module have a smaller period, such that $\lambda_{n+1}=s \lambda_{n}$, where $s<1$ is a constant contraction factor. The set of spatial periods forms a geometric sequence. Such grid codes consist of nested intervals by design and are unsuited for modular arithmetic.

- Stochastic ensembles. For $N$ cells, a divisor $L \mid N$ is chosen randomly. Then the spatial periods are drawn identically from one of two distributions: in the first case, from the uniform distribution [0,1]; in the second case, from the uniform distribution $[(1-A) \cdot \mathrm{s}, A+$ $(1-A) \cdot \mathrm{s}]$, where $s$ is a random shift variable and $A$ a random amplitude, both drawn uniformly from $[0,1]$. Thereby $70 \%$ of the realizations were drawn from $[0,1]$ (first case). The second case results in more densely spaced spatial periods, all of which lie within $\pm(1-A) \cdot \mathrm{s}$ of the period with length $A$, which tends to favor decoding based on modular arithmetic. In general, drawing from the stochastic ensemble can yield spatial periods that fit either the nested interval or a modular arithmetic scheme. The resulting grids embody generic modular codes consisting of periodically spaced tuning curve peaks.

The choice of spatial periods for the grid affects both the range and the resolution of the code. In the absence of noise, a well-designed grid code could simultaneously span large distances and discriminate fine differences in position; however, intrinsic variability introduces trade-offs between these two properties of the code. While the modular arithmetic scheme does not require closely spaced spatial periods a priori, the close spacing becomes important in the presence of noise. Hence, the nested interval and the modular arithmetic schemes become distinct if one insists that the spatial range in the latter scheme be robust. We now submit both schemes to the crucial test: Can one reliably estimate the position by counting the spikes from a finite set of neurons within a limited time window? We start by contrasting the resolution of grid and place codes for populations of neurons.

\section{Population Coding Model}

We consider a population of $N$ stochastically independent Poisson neurons (similar to Paradiso, 1988; Seung \& Sompolinsky, 1993; Salinas \& Abbott, 1994; Bethge et al., 2002; Pouget, Dayan, \& Zemel, 2003; Huys, Zemel, Natarajan, \& Dayan, 2007, for instance). The firing rate of each neuron depends on the one-dimensional position $x$ on the unit interval $X=[0,1]$. A priori, each position is equally likely, resulting in a flat prior $P(x)=1$.

The firing rate of neuron $i$ is described by its tuning curve $\left\{\alpha_{i}(x)\right\}_{i \leq N}$. Given a position $x \in[0,1]$, the conditional probability of observing the $N$-dimensional spike pattern $K=\left(k_{1}, \ldots, k_{N}\right) \in \mathbb{N}^{N}$ in a time interval of 
length $T$ is

$$
P(K \mid x)=\prod_{i \leq N} \operatorname{Poisson}\left(k_{i}, T \cdot \alpha_{i}(x)\right)=\prod_{i \leq N} \frac{\left(T \cdot \alpha_{i}(x)\right)^{k}}{k !} \cdot \exp \left(-T \cdot \alpha_{i}(x)\right) .
$$

The maximal firing rate $f_{\max }=\max _{x \in X, i \leq N}\left(\alpha_{i}(x)\right)$ is assumed to be constant across the population. Periodic tuning curves $\alpha_{i}(x)$ correspond to grid codes, whereas single-peaked, aperiodic $\alpha_{i}(x)$ correspond to place codes.

The tuning curves of place cells are taken as gaussian functions with centers distributed equidistantly over $X=[0,1]$ :

$$
\alpha_{i}(x)=f_{\max } \cdot \exp \left(-\frac{\left(x-\frac{i}{N-1}\right)^{2}}{2 \sigma^{2}}\right) \text { with } 0 \leq i<N
$$

The free parameters are the maximal firing rate $f_{\max }$, the tuning width $\sigma$, and the number of neurons $N$. Figure 3 a illustrates this family of tuning curves for $N=12$ cells with tuning width $\sigma=0.1$.

In contrast, the tuning curves for grid cells are defined as periodic functions with gaussian-like bumps of the type $\exp \left(-\frac{\left(-\frac{\lambda}{2}+\bmod \left(\frac{\lambda}{2}+x, \lambda\right)\right)^{2}}{2 \sigma^{2}}\right)$. Here $\bmod (z, \lambda)$ stands for the remainder after dividing $z$ by the spatial period $\lambda$.

To construct a family of grid cell tuning curves, we vary the spatial periods and the spatial phases. Each spatial period $\left\{\lambda_{l}\right\}_{l<L}$ defines a grid module; each of the $L$ modules contains $M=N / L$ equidistantly arranged phases within its periodic domain. Hence, for each module $\lambda_{i}$, with $0 \leq i<$ $L$, there are equidistant spatial phases $\varphi_{j}=\frac{j \cdot \lambda_{i}}{M}, 0 \leq j<M$, per module $\lambda_{i}$ and tuning curves:

$$
\alpha_{i, j}(x)=f_{\max } \cdot \exp \left(-\frac{\left(-\frac{\lambda_{i}}{2}+\bmod \left(\frac{\lambda_{i}}{2}+x-\varphi_{j}, \lambda_{i}\right)\right)^{2}}{2 \sigma_{i}^{2}}\right) .
$$

Figure $3 b$ illustrates a grid code for 12 cells with two spatial periods. After fixing $f_{\max }$ and $N$, the only remaining free parameter for the place code is the spatial tuning width $\sigma$, whereas for the grid code, the set of spatial periods $\left\{\lambda_{1}, \ldots, \lambda_{L}\right\}$ needs to be specified.

Both coding schemes should enable real-time readout of the rat's position while it is moving. During active exploration of the environment, $7 \mathrm{~Hz}$ to $12 \mathrm{~Hz}$ theta oscillations course through the parahippocampal loop, acting as a Zeitgeber (Buzsaki, 2006). Within this natural time frame of roughly $T=80-140 \mathrm{~ms}$, the maximal expected spike count of a grid or place cell is generally low. With measured peak firing rates of place and grid fields 


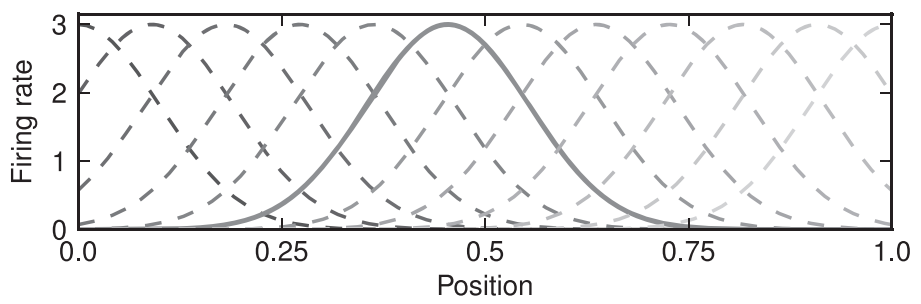

(a)
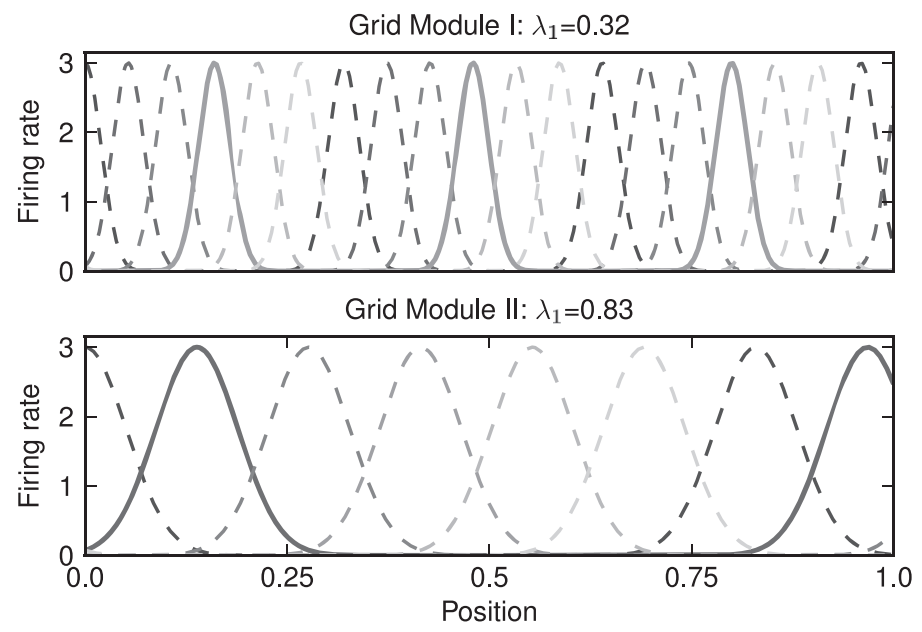

(b)

Figure 3: Families of tuning curves. (a) Family of place cell tuning curves: Different grayscales represent different cells. Tuning curves of 12 place cells with $\sigma=0.1$ and maximum firing rate $f_{\max }=3$. (b) Family of grid cell tuning curves for two different spatial periods. Different grayscales represent different cells. Tuning curves of 12 grid cells with spatial periods $\lambda_{1}=0.32$ and $\lambda_{2}=0.83$, 6 phases per module, and maximum firing rate $f_{\max }=3$. In this model, the tuning width of the grid cells scales with the spatial period, as suggested by experimental data (Brun et al., 2008).

in the range of $10 \mathrm{~Hz}$ to $30 \mathrm{~Hz}$ (Hafting et al., 2005; Leutgeb, Leutgeb, Treves, Moser, \& Moser, 2004), $f_{\max } \cdot T \approx 1-4$ within one theta cycle. For our analysis, we choose $f_{\max } \cdot T=3$.

By observing the spike counts from a population of $N$ neurons, one can build an estimator $\hat{x}$ of the position $x$. The average mean square error is (Salinas \& Abbott, 1994; Bethge et al., 2002)

$$
\chi^{2}=\mathbb{E}\left((x-\hat{x})^{2}\right)=\sum_{K \in \mathbb{N}^{N}} \int_{0}^{1}(x-\hat{x}(K))^{2} \cdot P(K \mid x) p(x) d x
$$


The $\chi^{2}$ error generally depends on the estimator $\hat{x}$. For instance, given a particular population response $K$, the most likely stimulus that gave rise to it is

$$
\hat{x}_{\mathrm{MLE}}(K)=\max _{x \in[0,1]} P(x \mid K)
$$

which is known as the maximum likelihood estimate (MLE). The corresponding mean maximum likelihood estimate square error (MMLE) is defined as $\chi_{M L E}^{2}=\mathbb{E}\left(\left(x-\hat{x}_{M L E}\right)^{2}\right)$. We compute the MMLE as described in the appendix by Monte Carlo methods. As the MMLE is numerically expensive to compute for large population sizes, we compare it against a reference that can be computed analytically. The MLE is both statistically efficient and consistent (Lehmann \& Casella, 1998), which means that $\chi_{M L E}^{2}$ asymptotically approaches the mean asymptotic square error (AE) for an increasing number of independent and identically distributed (i.i.d.) observations:

$$
\chi_{A E}^{2}=\mathbb{E}(1 / J(x)),
$$

as shown in Bethge et al. (2002). Here $J(x)$ stands for the Fisher information:

$$
J(x)=\mathbb{E}\left(\left(\frac{\partial}{\partial x} \ln (P(K \mid x))\right)^{2}\right) .
$$

However, for low numbers of spikes and a single observation and low spike counts, the true $\chi^{2}$ can exceed $\chi_{A E}^{2}$, as reported by Bethge et al. (2002) for the minimum mean square error. For this reason, we employ $\chi_{A E}^{2}$ as a practical benchmark that gives us scaling rules for grid and place codes. We then investigate how a population code can be cleverly constructed so that maximum likelihood decoding of the population response comes close to this benchmark, even for low, realistic spike counts.

The Fisher information, equation 3.7, for a population model with independent, Poisson neurons has a simple structure (compare Paradiso, 1988; Seung \& Sompolinsky, 1993, for instance):

$$
J_{\text {Population model }}(x)=\sum_{i=1}^{N} \frac{T\left(\partial \alpha_{i}(x) / \partial x\right)^{2}}{\alpha_{i}(x)}
$$

so that the asymptotic error is

$$
\chi_{A E}^{2}=\int_{0}^{1}\left(\sum_{i \leq N} \frac{T\left(\partial \alpha_{i}(x) / \partial x\right)^{2}}{\alpha_{i}(x)}\right)^{-1} d x
$$


This quantity depends only on the tuning curves and their first derivatives. Following the tradition of comparing neuronal codes on the basis of the Fisher information (Zhang \& Sejnowski, 1999; Eurich \& Wilke, 2000; Wilke \& Eurich, 2002; Brown \& Bäcker, 2006), we ask: Based on the error measures $\chi_{M L E}^{2}$ and $\chi_{A E}^{2}$, can a grid code outperform a place code? In particular, which spatial periods should be present in the grid code? What should the width of the firing field be relative to the spatial period?

3.1 Average Fisher Information and Asymptotic Resolution. For the families of place code (PC) and grid code (GC) tuning curves defined by equations 3.2 and 3.3, the Fisher information, equation 3.7, of a single cell is given by

$$
J_{P C, i}(x)=T f_{\max } \cdot \frac{\left(x-\frac{i}{N-1}\right)^{2}}{\sigma^{4}} \cdot \exp \left(-\frac{\left(x-\frac{i}{N-1}\right)^{2}}{2 \sigma^{2}}\right)
$$

and

$$
\begin{aligned}
J_{G C, i j}\left(x, \varphi_{j}\right)= & T f_{\max } \cdot \frac{\left(-\frac{\lambda_{i}}{2}+\bmod \left(\frac{\lambda_{i}}{2}+x-\varphi_{j}, \lambda_{i}\right)\right)^{2}}{\sigma_{i}^{4}} \\
& \cdot \exp \left(-\frac{\left(-\frac{\lambda_{i}}{2}+\bmod \left(\frac{\lambda_{i}}{2}+x-\varphi_{j}, \lambda_{i}\right)\right)^{2}}{2 \sigma_{i}^{2}}\right) .
\end{aligned}
$$

For the Fisher information of a population of cells, with $J(x)=\sum_{i j} J_{i j}(x)$, Jensen's inequality implies

$$
\chi_{A E}^{2}=\int_{0}^{1} \frac{1}{J(x)} d x \geq \frac{1}{\int_{0}^{1} J(x) d x}
$$

The closer $J(x)$ comes to being a constant, so that it is independent of the position $x$, the tighter the inequality is. Therefore, the asymptotic error is easy to calculate in the following limits: for place codes, when the equidistant tuning curves tile the full range densely, or, for grid codes, when the phase-shifted tuning curves tile each spatial period densely.

In these limits, the Fisher information for the population approaches $N$ times the average Fisher information per cell. The asymptotic error is simply the inverse of the Fisher information conveyed by the population. For the place code, we first compute the average Fisher information for a tuning 
curve centered at $c \in[0,1]$ and then average over all possible centers $c$ :

$$
\begin{aligned}
\overline{J_{P C}} & =\int_{0}^{1} \int_{0}^{1} f_{\max } T \cdot \frac{(x-c)^{2}}{\sigma^{4}} \exp \left(-\frac{(x-c)^{2}}{2 \sigma^{2}}\right) d x d c . \\
& =f_{\max } T \cdot\left(\frac{\sqrt{2 \pi}}{\sigma} \operatorname{erf}\left(\frac{1}{\sqrt{2} \sigma}\right)+4 \cdot \exp \left(-\frac{1}{2 \sigma^{2}}\right)-4\right) \\
& \propto \frac{\sqrt{2 \pi} f_{\max } T}{\sigma} \quad \text { for } \sigma \ll 1 .
\end{aligned}
$$

This result (Brown \& Bäcker, 2006) shows that the average Fisher information of one place cell is inversely proportional to the tuning width $\sigma$-the narrower the tuning curve, the better (see Figure 4a); this finding coincides with the result for stimuli that are not restricted to a compact subset of $\mathbb{R}$ (Zhang \& Sejnowski, 1999). If the tuning curves for place cells cover the span $[0,1]$ sufficiently densely and uniformly, then the resolution of the place code, as measured by the MLE, will approach the Cramér-Rao bound $\left(N \cdot \overline{J_{P C}}\right)^{-1}$. For fixed $N$, the tuning width cannot be reduced indefinitely while maintaining uniform coverage of the unit interval. Indeed, for fixed $N$ and for any $\epsilon>0$, there will be a $\sigma(\epsilon)>0$ and subintervals $K \subset[0,1]$ of fixed length $l$, such that for all $\sigma<\sigma(\epsilon)$ and $x \in K: J_{P C, N}(x)<\epsilon$. By Jensen's inequality, equation $3.12, \chi_{A E}^{2}=\geq \frac{1}{\epsilon}$, and hence $\chi_{A E}^{2}(P C, N) \rightarrow \infty$ for $\sigma \rightarrow 0$. This means that there is an optimal $\sigma$ for finite ensembles. For instance, for $N=100$, the smallest asymptotic error is attained for $\sigma \approx 4.1 \cdot 10^{-3}$, leading to a resolution of $\chi_{A E}^{2} \approx 6 \cdot 10^{-6}$. This value is used as a benchmark for comparison with grid codes.

In general, a population of place cells will have

$$
\overline{J_{P C, N}} \propto f_{\max } T \cdot \sum_{i=1}^{N} \frac{1}{\sigma_{i}} \approx f_{\max } T \cdot N\left\langle\frac{1}{\sigma_{i}}\right\rangle
$$

if we do not assume that all tuning curves have equal width. In some cases, place cells have multiple peaks, although the average number of peaks is close to one (Leutgeb et al., 2007; Fenton et al., 2008). If there are $\gamma$ peaks per place cell and the tuning widths are optimized, then the Fisher information at best scales as $\left(\gamma N^{2}\right)$ in the number of neurons. If the tuning widths are not simultaneously scaled, in contrast, the Fisher information scales linearly in $N$.

By comparison, the spatial map of a grid cell has multiple bumps, by definition. If the Fisher information for each bump scales as $\sigma^{-1}$, just as in a place cell, and there are $\lambda^{-1}$ bumps in the unit interval, then the mean Fisher information in a grid cell scales as $(\lambda \sigma)^{-1}$. This is indeed correct, as the following more formal argument shows. For the mean Fisher information of a grid cell, we have to average the Fisher information, equation 3.11, over 


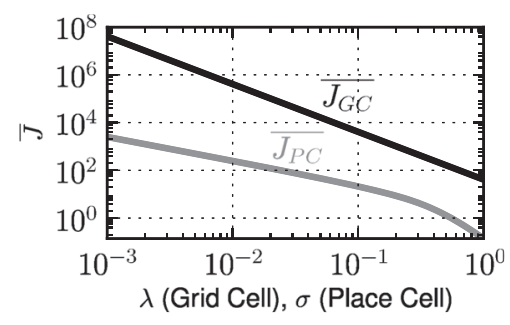

(a)

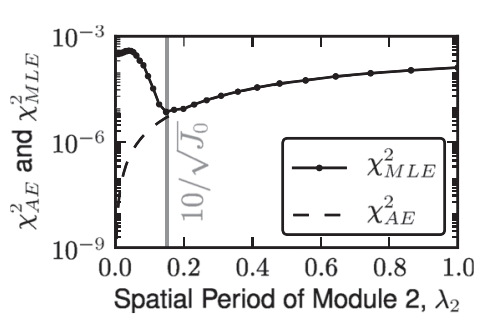

(b)

Figure 4: (a) Average Fisher information versus spatial period $\lambda$ and tuning width $\sigma$, both normalized to the unit span $[0,1]$. The average Fisher information of a grid cell $\overline{J_{G C}}$ scales as $\lambda^{-2}$, whereas the average Fisher information of a place cell $\overline{J_{P C}}$ scales as $\sigma^{-1}$. For $\sigma \approx 1$, the tuning curve becomes wider than the stimulus space, leading to a more rapid fall-off in the average Fisher information of the place cell than $\sigma^{-1}$. (b) Mean maximum likelihood estimate square error $\chi_{M L E}^{2}$ and mean asymptotic square error $\chi_{A E}^{2}$ for a grid code on a one-dimensional unit interval with two modules of $M=25$ neurons each. We use Monte Carlo methods to compute $\chi_{M L E}^{2}$, whereas the analytical Fisher information is used for the asymptotic estimate. The first module is nonperiodic and comprises 25 equidistantly arranged gaussian tuning curves with tuning width $\sigma=1 /(5 \sqrt{2})$ and a $10 \mathrm{~Hz}$ peak firing rate, integrated over $T=1$ second. This corresponds to a peak spike count of 10 , much larger than $f_{\max } \cdot T \approx 1$ in Bethge et al. (2002). The second module also comprises 25 equidistantly arranged cells with tuning curves that are periodically extended versions of the tuning curves of the first module with spatial period $\lambda_{2}$. The numerically determined $\chi_{M L E}^{2}$ closely follows the asymptotic error given by the inverse Fisher information $\chi_{A E}^{2}$ for spatial periods of $\lambda_{2}>0.18$. This is roughly $10 \cdot 1 / \sqrt{J_{0}}$, that is, 10 times the square root of the inverse Fisher information of module 1 . If the periodicity of the next module falls below the typical range of errors made by the first module, the Fisher information ceases to capture the MLE error.

all possible spatial phases $\varphi$. Due to periodicity, it suffices to average over phases from 0 to the spatial period $\lambda$ :

$$
\overline{J_{G C}}=\frac{1}{\lambda} \cdot \int_{0}^{\lambda} \int_{0}^{1} J_{G C}(x, \varphi) d x d \varphi .
$$

For $\lambda \ll 1, \int_{0}^{1} J_{G C}(x, \varphi) d x \approx \frac{2}{\lambda} \cdot \int_{0}^{\frac{\lambda}{2}} J_{G C}(x, 0) d x$, because of the periodicity of $J_{G C}(x, \varphi)$ in $x$. Hence,

$$
\begin{aligned}
\overline{J_{G C}} \approx & \frac{2 f_{\max } T}{\lambda} \int_{0}^{\lambda / 2} \frac{x^{2}}{\sigma^{4}} \exp \left(-\frac{x^{2}}{2 \sigma^{2}}\right) d x \\
& \sim \sqrt{2 \pi} \frac{f_{\max } T}{\lambda \sigma},
\end{aligned}
$$


for $\sigma \ll \lambda$. The derivation of the exact formula is given in the appendix. The tuning width $\sigma$ can be expressed as a product of the spatial period $\lambda$ and the relative tuning width per spatial period, which we call the area ratio $r_{A}$. We define the tuning curve's width by the firing rate relative to the maximum firing rate. If $f \geq \beta f_{\max }$ delineates a firing field, then the following relationship holds:

$$
\sigma=\frac{r_{A} \cdot \lambda}{2 \sqrt{\log \left(1 / \beta^{2}\right)}} .
$$

Consequently, we have $\overline{J_{G C}} \sim \frac{f_{\max } T}{\lambda^{2} r_{A}}$. In Figure 4a, the average Fisher information of a grid and a place cell is compared. Both parameters, the spatial period and the tuning width, are expressed in terms of the normalized stimulus range and are varied between 0 and 1 . Whereas the average Fisher information of a place cell is inversely proportional to the tuning width $\sigma$, the average Fisher information of a grid cell is inversely proportional to the square of the spatial period $\lambda$. As the tuning curve width $\sigma$ narrows, the mean firing rate in a place code decreases, whereas a grid cell maintains a constant mean firing rate as $\lambda$ changes, by construction. On a per spike basis, the scaling of the average Fisher information with $\sigma$ is identical for place cells and grid cells.

By rescaling the lattice length scale $\lambda$, the local resolution of a grid cell population can improve. Yet periodicity also introduces ambiguity, such that a typical neuronal response for a single grid cell maps onto $\lfloor 2 / \lambda\rfloor$ possible values of $x$. Adding neurons with shifted tuning curves of the same spatial period and considering the population response still leads to ambiguity. So the error made in decoding can be large, even though the Fisher information indicates otherwise. Indeed, for $\lambda \ll 1$, the expected error approaches the variance of $x$ over the uniform distribution on the interval $[0,1]$ :

$$
\chi_{A E}^{2}=1 / \overline{J_{G C}} \sim 1 / \lambda^{2} \quad \underset{\lambda \rightarrow 0}{\ll} \mathbb{E}\left((x-\hat{x})^{2}\right)=1 / 12
$$

Hence, $\chi_{A E}^{2}$ can be much less than $\chi_{M L E}^{2}$, for instance; the asymptotic estimate falls far short of what can realistically be achieved using any decoder. The solution lies in using different length scales in parallel, which allows one to exploit the higher resolution at short length scales. This observation also emphasizes that the MASE analysis has to be supplemented by numerical studies of the MMLE for grid codes.

3.2 Modular Codes, Self-Similarity, and Power Law Scaling. As pointed out above, the asymptotic error (AE) may never be achieved by maximum likelihood estimation (MLE) or any other estimator, as a grid code's periodicity causes ambiguity, even in the absence of noise: if we consider 
the population response as a code word, there will be distinct stimuli that give rise to the same code word. Therefore, we now construct a class of grid codes, called nested grid codes, that will contain no recurring codewords for stimuli on the interval $[0,1]$. For such codes, MLE can attain the asymptotic error, as we show later.

A nested modular code consists of dividing the population of $N$ neurons into $L$ subgroups of $M_{i}$ neurons, whose tuning curves are periodic on the same length scale $\lambda_{i}$. Each subgroup is called a module. The range of stimuli that such a nested grid code represents is at least as long as the longest lattice length scale $\max \left(\lambda_{i}\right)$, and possibly much longer. But for simplicity, take $\max \left(\lambda_{i}\right)>1$, as some of the ideal modular grid codes with optimal resolution derived below will have a range that is exactly $\max \left(\lambda_{i}\right)$. Furthermore, we make the a priori assumption that each module can be read out individually, i.e., that a spatial phase relative to the length scale $\lambda_{i}$ can be determined from the population response of this module. According to equation 3.17, the Fisher information of a given module scales as

$$
\bar{J}_{i} \sim \frac{M_{i}}{\lambda_{i}\left\langle\sigma_{i}\right\rangle},
$$

in which $\left\langle\sigma_{i}\right\rangle$ is the average width parameter for the tuning curves in the module (see the appendix for the precise statements). Within one spatial period, the grid cells code position the same way place cells do. Hence, as is the case for place cells, the optimal tuning width scales as

$$
\left\langle\sigma_{i}\right\rangle \sim \frac{\lambda_{i}}{M_{i}}
$$

So the Fisher information for the module scales as

$$
\bar{J}_{i}=\frac{C_{1}^{2} M_{i}^{2}}{\lambda_{i}^{2}},
$$

when the tuning curve widths are optimized. Here $C_{1}^{2}$ is a constant, which we write using a power of two for later convenience. Summing over all modules, the Fisher information of the grid code can be written as

$$
\overline{J_{G C, N}}=C_{1}^{2} \sum_{0 \leq i \leq L-1} \frac{M_{i}^{2}}{\lambda_{i}^{2}} .
$$

Within any grid code, the spatial periods can always be ordered so that $\lambda_{0}>\lambda_{1}>\cdots>\lambda_{L-1}$. In a nested grid scheme, two types of error can occur during decoding. Imagine a grid code with two modules and periods $\lambda_{0}>$ $\lambda_{1}$. The module with the shorter spatial scale $\lambda_{1}$ refines the representation at the coarser scale $\lambda_{0}$, such that the period $\lambda_{1}$ "discretizes" the period $\lambda_{0}$ (note 
that we do not assume that $\lambda_{0}$ is an integer multiple of $\left.\lambda_{1}\right)$. If $\hat{x}$ is an estimate of the position $x$ based on module $\lambda_{0}$, then there is a finite probability that $|\hat{x}-x|>\lambda_{1}$. In such an event, which we call a discretization error, the module with period $\lambda_{1}$ cannot improve the estimate of $x$. The second type of error is the local error, which is less catastrophic and is bounded by the inverse of the Fisher information.

To limit the probability of a discretization error per module to less than $\epsilon$, we will insist that

$$
D(\epsilon) / \sqrt{\bar{J}_{i}} \leq \lambda_{i+1} \leq \lambda_{i},
$$

where $D(\epsilon)$ is a safety factor. This safety factor can be computed from the probability distribution of the deviation between the (efficient) estimate $\hat{x}$ and the true value $x$, based on the population spike count from a single module. In the asymptotic limit $\left(M_{i} \gg 1\right.$ and $\left.f_{\max } T \gg 1\right)$, this probability distribution can be modeled by the Laplace approximation $p(x-\hat{x}) \propto \exp \left[-(x-\hat{x})^{2} \bar{J}_{i} / 2\right] ;$ hence,

$$
D(\epsilon)=\sqrt{2} \operatorname{erfc}^{-1}(\epsilon) .
$$

For instance, a safety factor $D(\epsilon)=4$ guarantees that the discretization error probability is less than $10^{-4}$. Given such a constraint, the Fisher information, equation 3.23 , is maximized when the lower bound in equation 3.24 is attained. This implies that

$$
\lambda_{i}=\lambda_{0} \cdot\left(\prod_{j<i} \frac{C_{1}}{D(\epsilon)} M_{j}\right)^{-1} .
$$

Defining $\widetilde{M}_{j}=\sqrt{C_{1} / D(\epsilon)} M_{j}$, the population Fisher information, equation 3.23 , becomes

$$
\overline{J_{G C, N}}=\frac{C_{1} D(\epsilon)}{\lambda_{0}^{2}} \sum_{0 \leq i \leq L-1} \prod_{j \leq i} \tilde{M}_{j}^{2}
$$

Maximizing the Fisher information in equation 3.27 for integer $M_{i}$ subject to the constraint $\sum_{i=0}^{L-1} M_{i}=N$ leads to

$$
M_{i} \approx N / L,
$$

as long as $L \ll \sqrt{C_{1} / D(\epsilon)} N$. For instance, if $\sqrt{C_{1} / D(\epsilon)} \sim \mathcal{O}(1)$, then the condition for $M_{i} \approx N / L$ reads $N / L \geq 3$. Otherwise, $M_{i}=3$ for $i \leq\lfloor N / 3\rfloor$ and 
$M_{i}=0$ for $i>\lceil N / 3\rceil$ leads to the maximal Fisher information. Therefore, we should assign an equal number $M$ of grid cells to each grid module, so that all modules are self-similar. As a corollary, the area ratio $r_{A}$ between mean field width and the spatial period should be constant across modules. This prediction is consistent with experimental data from Brun et al. (2008). The experimentally determined ratio of field width to period is $r_{A} \approx 0.3$. $^{2}$ This ratio remains approximately constant along the dorso-ventral axis of $\mathrm{mEC}$ even as the spatial period $\lambda$ varies.

For constant $M$, equation 3.24 indicates that the sequence of length scales $\lambda_{i}$ should form a geometric progression. In this case, the population's Fisher information becomes

$$
\begin{aligned}
\overline{J_{G C, N}} & =\frac{M^{2} C_{1}^{2}}{\lambda_{0}^{2}} \cdot \sum_{i=0}^{L-1} \tilde{M}^{i}=\frac{M^{2} C_{1}^{2}}{\lambda_{0}^{2}} \frac{\tilde{M}^{2 L}-1}{\tilde{M}^{2}-1} \\
& \geq \frac{C_{1} D(\epsilon)}{\lambda_{0}^{2}}\left(\tilde{M}^{2}\right)^{N / M} .
\end{aligned}
$$

Hence, the Fisher information for a nested grid code obeys a power law in the number of neurons $N$ for fixed module size $M$. Such a coding scheme therefore outperforms a place code that scales at best as $N^{2}$, which happens when the tuning width scales as $N^{-1}$.

We need to resort to numerical simulations to test whether $\overline{J_{G C, N}}$, as given by equation 3.29 , reliably predicts the true error in decoding $x$ from the neuronal response measured over short time windows. Figure $4 \mathrm{~b}$ reveals that the error in the maximum likelihood estimate is close to the asymptotic error as long as the safety factor $D(\epsilon)$ is sufficiently large.

In summary, for a modular grid code to achieve high spatial resolution, the grid lattices should form a geometric progression in the spatial periods, and each module should be self-similar. Only relatively few distinct spatial phases are needed at each length scale, but they should generally number at least three. If the number of encoded phases is low, the spatial tuning width should be broad to ensure that the animal's position is uniformly and isotropically represented, even when observing only a finite subset of neurons.

\section{The Spatial Resolution of Maximum Likelihood Decoding}

Within a fixed time window $T$, neurons will fire a finite number of spikes, yielding a population vector $K$ of spike counts. As the animal moves, this time window needs to be short to create a running estimate $x$, which will

\footnotetext{
${ }^{2}$ Experimentally defined as the median of the set of pairwise grid field to grid field spacings.
} 


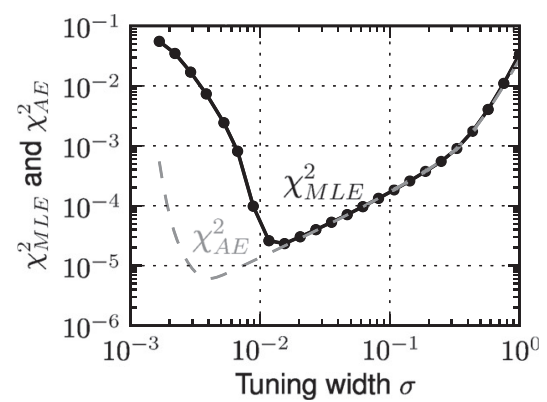

(a)



(b)

Figure 5: Mean maximum likelihood estimate square error $\left(\chi_{M L E}^{2}\right)$ and mean asymptotic square error $\left(\chi_{A E}^{2}\right)$ of place codes and of nested grid codes with 100 neurons, $f_{\max } T=3$. (a) Double logarithmic plot of the mean maximum likelihood estimator square error $\chi_{M L E}^{2}$ as a function of the spatial width $\sigma$ compared with the mean asymptotic square error $\chi_{A E}^{2}$ for place code comprising 100 cells and $f_{\max } T=3$. (b) The mean maximum likelihood estimate square error $\chi_{M L E}^{2}$ for geometric progressions of grid lattice spacings with contraction factor $s$, compared to the mean asymptotic square error $\chi_{A E}^{2}$. The factor $s$ determines the spatial periods as $\lambda_{i}=s^{i-1}$ for $1 \leq i \leq 10$. Each module comprises 10 equidistantly arranged spatial phases.

rely only on a few spikes. Maximum likelihood (ML) decoding requires performing numerical calculations (see the appendix) and returns the most likely position $x$ given $K$. Such estimates will be subject to both local and global errors; the Fisher information predicts only the local error in the limit as $f_{\max } T \rightarrow \infty$. Therefore, the ML error $\chi_{M L E}^{2}$ may diverge from the asymptotic error $\chi_{A E}^{2}$, and the optimal parameter settings will change. We will use ML to study both grid codes for which the spatial periods are asymptotically optimal and grid codes drawn from random ensembles. Randomly selecting the spatial periods will reveal how generic the properties of good grid codes are.

4.1 Maximum Likelihood Decoding: Simulation Results. We calculated the spatial resolution by maximum likelihood methods, again for a population of 100 grid and place cells, respectively, and $f_{\max } T=3$. To examine the error made in reading out the place code, we varied the width $\sigma$ of the tuning curves.

The simulations show that the mean maximum likelihood error $\left(\chi_{M L E}^{2}\right)$ of a place cell diverges substantially from the mean asymptotic square error $\left(\chi_{A E}^{2}\right)$ for small tuning widths sigma, that is, for narrow place fields (see Figure 5a). In particular, the spatial width that minimizes the asymptotic error is 10 times smaller than the width that minimizes the MLE. 
The grid codes differ not in the relative tuning width of the spatial firing rate profiles, but in the number of spatial periods and the length scales that describe the grid lattice spacing. Asymptotic theory (see section 3.2) predicts that these length scales should form a geometric sequence. By choosing the largest spatial period $\lambda_{1}$ to be unity and then creating grid codes characterized by different ratios for the successive periods, we investigate the concordance between the maximum likelihood error (MMLE) and the asymptotic error (see Figure 5). If the modules are nested so that the contraction factor $0.5<s<1$, the MLE approaches the asymptotic error. For factors $s<0.5$, the MMLE exceeds the asymptotic error; the asymptotic error keeps decreasing forever, whereas the MMLE will eventually increase. The MMLE is not convex, however, in $s$. When the contraction factor $s$ is close to an even divisor of unity, such as $s=1 / 2,1 / 3, \ldots$, the MMLE diverges more strongly from the asymptotic error. In such exceptional cases, all modules attain a maximum close to $x=1$, which, by the periodicity of the tuning curves, can be wrapped around to join the maximum at $x=0$. In these cases, positions close to the boundaries of the unit interval, i.e., close to either zero or one, elicit similar patterns of spikes. Mistaking a position $x=\epsilon$, where $\epsilon \ll 1$, for a position close to $1-\epsilon$, however, corresponds to a huge error. Hence, the MMLE is higher. Moreover, as the contraction factor becomes smaller, fewer intermediate modules remain. These modules with intermediate lattice spacings allow maximum likelihood estimation to correct for errors in the spatial phase represented by coarser modules. For $s \ll 1 / 2$, the increasing lack of compensation for errors causes the MMLE to rise, whereas the asymptotic error becomes ever smaller. Additionally, as $s \rightarrow 0$, any contraction factor becomes close to $1 / n$ for some $n$. These are the exceptional cases mentioned above that have high MMLE. Note that these exceptional cases can be avoided by taking $\lambda_{1}$ to be slightly larger than unity.

Hence, for grid codes whose modules are staggered in a geometric sequence, the resolution is much higher than in a place code (see Figure 5). Is this result generic? In other words, if one were to randomly put together a grid code with different spatial periods, would the resolution still be higher? To answer this question, we created randomly sampled grid codes as described in section 2, for which we estimated the MMLE. The histogram in Figure 6 shows the distribution of MMLEs for the ensemble. The grid codes' MMLE can then be compared to the MMLE for the optimal place coding scheme with the same number of neurons, depicted as a dashed reference line in Figure 6. Some grid codes are worse than the optimal place code: choosing a narrow span of spatial periods leads to poor spatial resolution (see the second highlighted example in Figure 6).

Closely spaced spatial periods should confer on the grid code the ability to uniquely represent an extended range of positions, going far beyond the unit interval (Fiete et al., 2008). Nonetheless, here we compare not the ranges of different grid codes but the ability of the codes to resolve positions within 


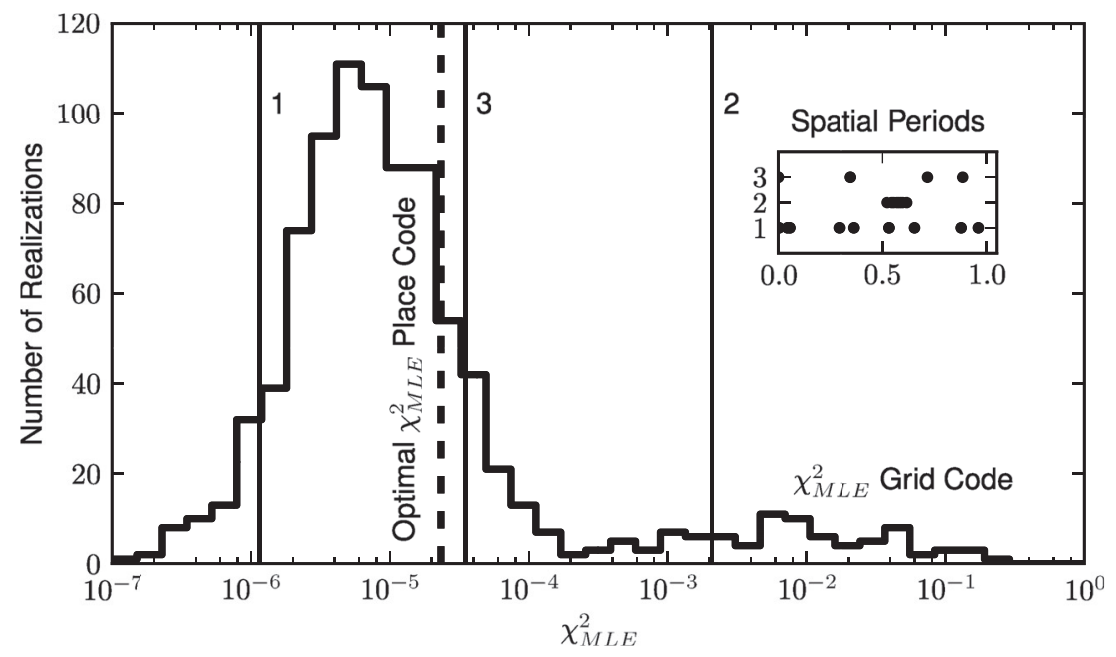

Figure 6: Histogram of mean maximum likelihood estimate square error $\left(\chi_{M L E}^{2}\right)$ for grid codes with 100 neurons, $f_{\max } T=3$. Histogram of MMLE for 885 simulated grid codes, which were randomly drawn according to the method described in section 2 and contrasted with the optimal place code MMLE displayed as a dashed line. The inset shows the spatial periods of the three example grid codes; the corresponding MMLE for these examples is marked on the histogram by a vertical line. Note that closely spaced spatial periods, such as in example 2, lead to poor spatial resolution.

the fixed unit interval. For some grid codes, the unit interval corresponds to only a fraction of the full theoretical range.

Around three-quarters of the randomly drawn grid codes have better MMLE than the best place code; hence, it is likely that a generic grid code, one with unrestricted range, will lead to a higher spatial resolution than the best place code.

What common properties do the better grid codes have? One key feature is that their spatial periods span a large range. For Figure 7, we binned the smallest and largest period of each grid code in the ensemble and depict the highest resolution for each binned pair of $\left(\min _{i} \lambda_{i}, \max _{i} \lambda_{i}\right)$. The resolution increases in both the direction of smaller $\min _{i} \lambda_{i}$ and, to a lesser degree, in the direction of larger $\max _{i} \lambda_{i}$. Each grid code is determined by the spatial periods of its modules. Figure 8 a depicts the set of spatial periods for the 10 best grid codes in the random ensemble. As suggested by the asymptotic analysis, the grid codes with the lowest MMLE have in common that the smallest spatial period, $\min _{i} \lambda_{i}$, is close to zero. In many cases, the largest spatial period, $\max _{i} \lambda_{i}$, nearly covers the entire unit interval represented by the code. The random sampling of spatial periods was unbiased: the a priori 


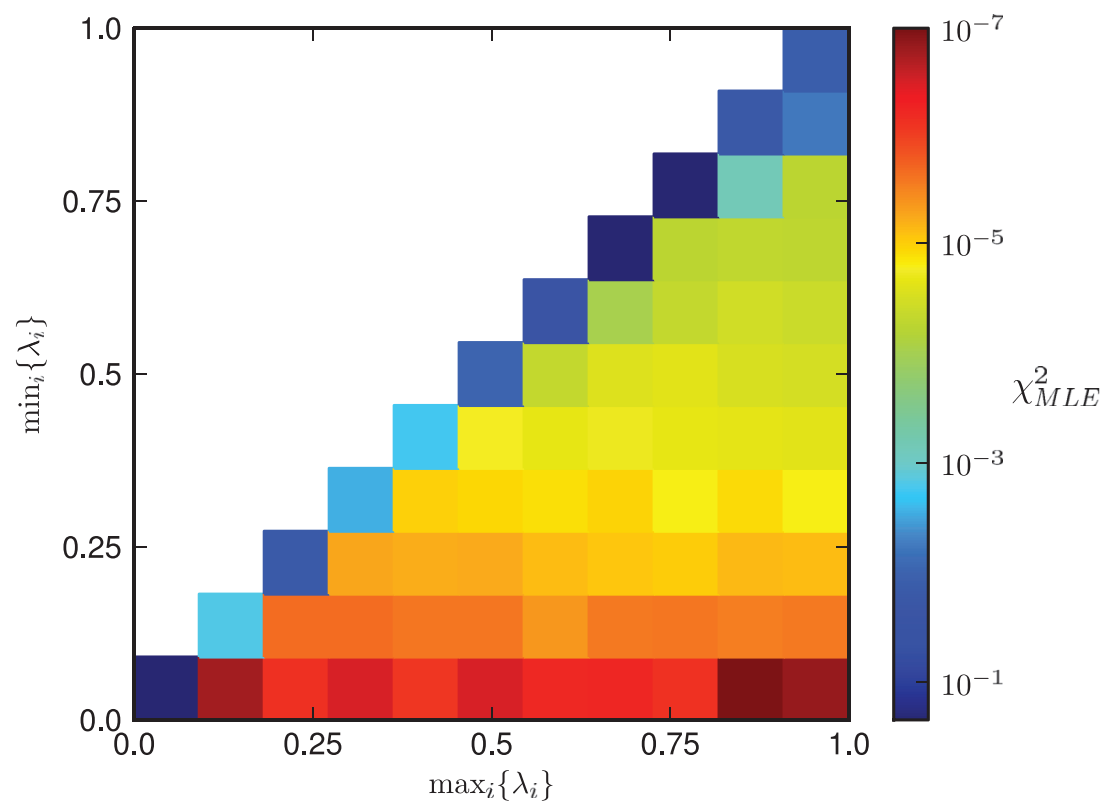

Figure 7: Mean maximum likelihood estimate square error $\left(\chi_{M L E}^{2}\right)$ as a function of the minimal and maximal spatial period. After dividing the spatial periods into bins, the smallest MMLE present in the random ensemble of grid codes is color-coded for each combination of smallest and largest spatial period. The results show that grid codes with similar smallest and largest spatial periods result in a large MMLE. Decreasing the smallest period while keeping the largest period fixed strongly improves the resolution; in contrast, keeping the smallest period fixed and increasing the largest period leads to a smaller improvement. The highest resolution is obtained when the smallest and largest period are far apart.

distribution of spatial periods is almost uniform (see Figure 8b). In the best grid codes, the smaller spatial periods are overrepresented. Selecting the 100 spatial periods from the best grid codes in the sample strongly shifts the distribution of spatial periods to the lower range (see Figure $8 b$ ).

Unlike the asymptotic error, which monotonically decreases with the smallest spatial period, the MMLE reaches an optimum. In the randomly sampled ensemble, going below $\min _{i} \lambda_{i} \approx 10^{-2}$ typically confers no advantage. A direct comparison between MMLE and the asymptotic error is shown in Figure 9. In some cases, the MMLE is much higher than the asymptotic error; throughout all cases, the MMLE never drops below $10^{-7}$ relative to the unit interval, whereas the asymptotic error can be orders of magnitude lower. One should note also that deterministically generating 

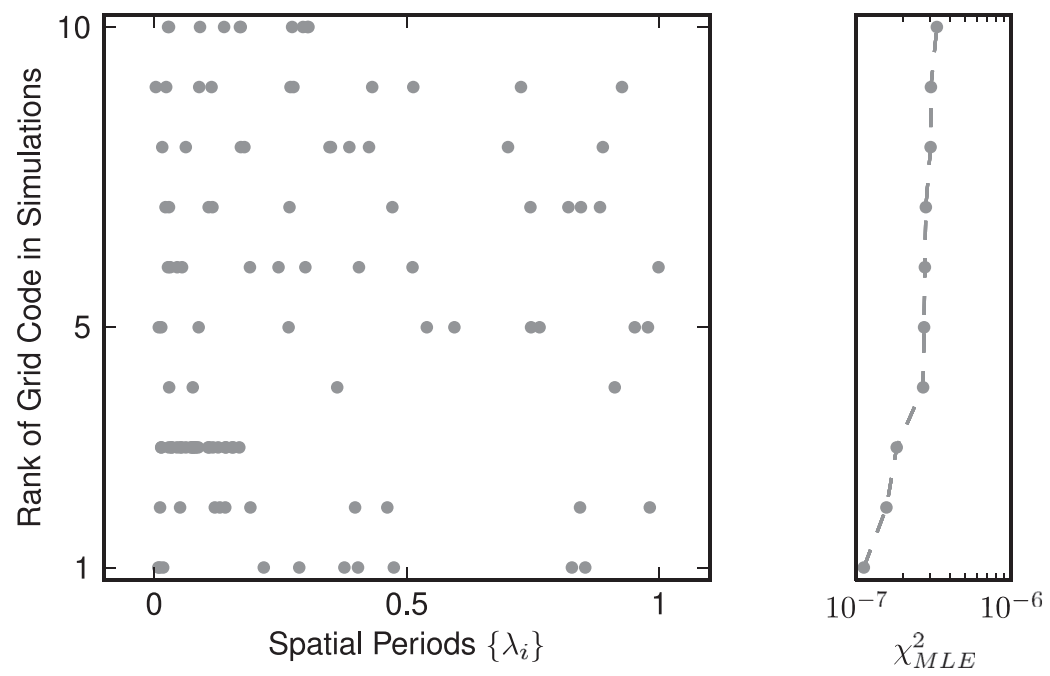

(a)

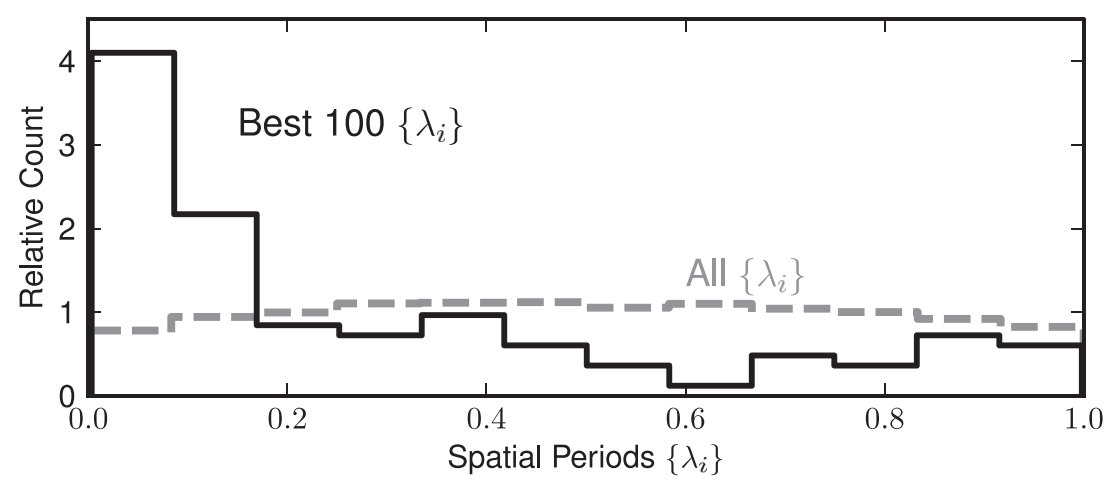

(b)

Figure 8: (a) Spatial periods of samples with highest mean maximum likelihood estimate square error $\left(\chi_{M L E}^{2}\right)$. Scatter plot of spatial periods of the 10 best grid codes in simulations and their corresponding MMLE, arranged from small to large MMLE. (b) Distribution of spatial periods with highest mean maximum likelihood estimate square error $\left(\chi_{M L E}^{2}\right)$. Histogram of the spatial periods in all simulated grid codes and the 100 samples with the lowest MMLE. The overall distribution has no substantial preference, whereas the distribution of the 100 spatial periods from the best grid codes is strongly skewed.

sequences of grid modules using equation 3.24 yields a considerably lower MMLE than even the lowest MMLEs in the random ensemble that we tested. 




Figure 9: Comparison of mean maximum likelihood estimate square error $\left(\chi_{M L E}^{2}\right)$ and mean asymptotic square error $\left(\chi_{A E}^{2}\right)$ for grid codes. Double logarithmic plot of MMLE versus asymptotic error for grid code plotted against the smallest spatial period. Smaller periods refine the unit interval more, yielding better spatial resolution. The asymptotic error decreases, on average, quadratically as the minimum spatial period becomes smaller, serving as a lower bound for the MMLE. Grid modules that are not properly nested lead to a much higher error than predicted asymptotically. Furthermore, the lower bound is no longer tight for $\min _{i} \lambda_{i}<10^{-2}$. No generic grid code from the random ensemble achieved an MMLE lower than $10^{-8}$, even though the asymptotic error values drop to $10^{-12}$.

\section{Discussion}

The neural representation of position in world coordinates is always subject to distortion due to the noisy, spiking nature of neurons. Just as photographing an athlete in motion rules out a long shutter time, capturing the instantaneous position as an animal explores its environment precludes averaging over long times - no matter whether single neurons fire at labeled positions (place cells) or at triangular lattice points in space (grid cells), noise will limit the resolution an animal needs to orient itself and navigate.

By considering stochastic models for neuronal populations, we have shown that grid cells can achieve higher spatial resolution than any possible arrangement of the same number of place cells. We computed the resolution for both coding schemes by decoding the most likely position in space from the number of spikes across the population within a short time window. 
The average divergence between the true and estimated position is bounded from below by the inverse of the average Fisher information, an analytically calculable measure of the asymptotic local coding precision: whereas the average Fisher information scales inversely with the tuning width for place cells, it scales inversely with the square of the tuning width for grid cells. Grid cells gain this advantage by firing at multiple locations in space; place cells, in contrast, inherently exhibit sparser neuronal discharge. But for a grid code to show improved spatial resolution over a place code, the grid lattices must be strategically arranged; many randomly constructed grid codes are actually worse than the best place codes.

Distortion theory predicts how well grid codes should be constructed. First, grid lattices should exist at different spatial scales, yet short-length scales should predominate. Each scale constitutes an independent module, comprising grid cells with a common spatial period $\lambda_{i}$ but different spatial phase offsets (Hafting et al., 2005, for instance). After constructing an ensemble of grid codes by randomly sampling $\lambda_{i}$, we found that good grid codes strongly skewed the distribution of $\lambda_{i}$ 's to small values, such that larger spatial periods are fewer yet still present: the full spatial range and the largest spatial period were typically of the same length scale and not an order of magnitude apart. Brun et al. (2008) recorded the spatial periods of different grid cells along the dorsoventral axis of the $\mathrm{mEC}$; the histogram of spatial periods is similar in its skew (Brun et al., 2008). Some grid cells had spatial periods of more than 8 meters on an 18 meter linear track. The typical lattice spacing of grid cells grows along the dorsoventral axis, yet reported grid cells were recorded along the first $75 \%$ of this axis, implying that longer-length scales may yet be found, particularly if it becomes feasible to record from rodents foraging on a football field. Our theoretical results also predict that the spatial periods should be plastic and adapt to the largest length scale in the local environment to achieve high spatial resolution. Indeed, grid lattices in $\mathrm{mEC}$ rescale when a familiar enclosure is artificially expanded or shrunk by a moderate factor, such that the relative positions of landmarks is maintained (Barry, Hayman, Burgess, \& Jeffery, 2007).

Second, achieving high spatial resolution with a fixed number of grid cells favors scaling the size of the firing fields with the spatial period of the grid module; furthermore, we can predict the ratio of firing field width to the spatial period. A grid module with spatial period $\lambda_{i}$ consists of several grid cells whose spatial lattices are shifted relative to each other. Hence, a grid code represents the spatial phase in firing field-sized bins, yielding a discretized phase.

If one distinguishes only whether a cell is active, one observes the following. Given $M$ grid cells that tile the range $[0,1)$ in a nonoverlapping manner, the phase resolution is at least $\Delta \varphi=1 / M$. If the next module recursively tiles each phase of the preceding module into $M$ bins, such a scheme would have a resolution of $\left(\frac{1}{M}\right)^{N / M}$, where $N$ is the number of cells. The highest 
spatial resolution is reached by trading off the number of spatial periods per module with the number of grid modules.

For discrete encoding, three grid cells per module are ideal, with the firing field of each grid cell covering one-third the spatial period. Each module associated with one spatial period will be perfectly nested inside another module. Nesting naturally gives rise to a strongly skewed distribution of spatial periods on a linear scale.

Some of the conclusions from the binary coding case considered above carry over to the continuous coding case, in which one discerns different firing rates. Maximizing the Fisher information of the population code reveals that the grid code should still stagger the modules' spatial periods in a geometric progression, $\lambda_{n+1}=s \lambda_{n}$. The contraction factor in the geometric series $s=\lambda_{i+1} / \lambda_{i}$ depends on the relative resolution of each module and hence crucially on the number of neurons per module and the peak firing rate. Because having more modules at the expense of phases per module is advantageous, the ratio of field width to spatial period should be comparatively large; in fact, the optimal ratio will approach the minimum allowed by the number $M$ of distinct phases. The ideal number $M$ is no longer necessarily three, but rather depends on the tolerable level of risk for catastrophic error during decoding. The greater $M$ is, the lesser this risk.

The design principles for grid codes were derived from asymptotic theory, which assumes that the time window for observing the neuronal population's response is sufficiently long. While the (asymptotic) Fisher information reveals how the error scales with tuning curve parameters (Zhang \& Sejnowski, 1999; Brown \& Bäcker, 2006), it could severely underestimate the true error (Bethge et al., 2002). We therefore pursued a systematic comparison between the asymptotic theory and the true maximum likelihood error, which was evaluated numerically by simulating the neuronal response over short time windows. For instance, one can construct a grid code with two modules for which the asymptotic error goes to zero as one lets the smallest spatial period become infinitely small. An analysis of the mean maximum likelihood error (MMLE), however, revealed that the minimal spatial period is in fact bounded. Likewise, the asymptotic error systematically underestimates the optimal tuning width for a place code. Yet the MMLE also confirmed some of the scaling properties of grid codes predicted by the Fisher information. For instance, the resolution of grid codes still scales exponentially in the number of neurons, implying that grid codes are superior to place codes, even under realistic conditions.

Our analysis suggests that even with noisy, spiking grid cells, the roughly $10^{5}$ neurons in the mEC (Mulders, West, \& Slomianka, 1997) should be able to encode the animal's position in space with exquisite precision. Four factors limit the effective resolution:

- The smallest spatial period cannot be arbitrarily small.

- Not all neurons in mEC contribute to encoding the position. 
- A realistic decoding mechanism will not achieve the resolution of an ideal observer.

- A putative decoder network may not have access to the whole ensemble of grid cells.

If we read out the spikes within one cycle $T$ of the ongoing theta oscillation while a rodent is running near its peak speed of about $150 \mathrm{~cm} / \mathrm{s}$ on a linear track, the minimal spatial period has to be bounded by $\lambda_{\text {min }}>T / v_{\text {max }} \approx 20 \mathrm{~cm}$. Otherwise the animal will traverse multiple grid lattice points within a single theta cycle. The spatial resolution for an ideal grid code scales with the square of the smallest period. Moreover, the spatial resolution will increase with the square root of the number of neurons that share this spatial period, but the effective number might be fewer than gross anatomy suggests. While place cells in the dentate gyrus and area CA3 of hippocampus are targets of layer II of $\mathrm{mEC}$, such neurons will presumably not be strongly connected to all neurons in $\mathrm{mEC}$ but to just a few. In general, a downstream neuron that decodes the animal's position might have access to only a restricted number of grid cell inputs; predicting the size of grid fields also required us to assume that the number of grid cells is finite. Several theoretical models propose that the ensemble firing of grid cells gives rise to single, isolated place fields in hippocampus by superposition (Fuhs \& Touretzky, 2006; Solstad et al., 2006; Rolls et al., 2006; Franzius et al., 2007; Si \& Treves, 2009; Cheng \& Loren, 2010); arbitrary or all-to-all connections between grid and place cell layers, however, often give rise to multiple firing fields (Solstad et al., 2006). The average of measured firing field to period ratios lies around 0.3 (Brun et al., 2008), which is consistent with both the theoretical prediction and the hypothesis that each place cell in DG and CA3 is strongly innervated by only a small subsample of grid cells from each grid module along the dorsoventral band (Solstad et al., 2006).

A key assumption in this analysis was that the spike counts obey a Poisson distribution. The fine temporal pattern of spike trains in both place and grid cells is anything but Poisson, as ongoing hippocampal-entorhinalcortical rhythms imprint their structure on the timing of spikes (Deshmukh, Yoganarasimha, Voicu, \& Knierim, 2010; Quilichini, Sirota, \& Buzsaki, 2010; Bragin et al., 1995). These rhythms might indeed be essential for generating the spatially localized firing fields in these cells (Burgess et al., 2007; Hasselmo, Giocomo, \& Zili, 2007; Burgess, 2008; Remme et al., 2010; Geisler et al., 2010). For instance, Geisler et al. correlate the frequency shift between intrinsic firing and the $7 \mathrm{~Hz}$ to $12 \mathrm{~Hz}$ theta oscillation in the local field potential with the size of the firing field in CA1 of hippocampus. Likewise, the spatial period and neural resonance properties correlate along the dorsoventral axis of the mEC (Garden, Dodson, O'Donnell, White, \& Nolan, 2008; Giocomo et al., 2007). We used the timescale of the theta oscillation to define the time window in which to count spikes but discount the 
fine structure of spike timing within this time window. Rapid oscillations largely average out in the sum that represents the probability of the spike count. The detailed temporal structure of hippocampal place cell firing can be captured by multiplying or linearly convolving the oscillations with the spatial tuning curve (Itskov, Pastalkova, Mizuseki, Buzsaki, \& Harris, 2008); repeated traversals of the firing field are accompanied by different phases of the oscillations, which adds to the variance of the spike count. Preliminary analysis of linear track data (Hafting et al., 2005) for grid cells indicates that the spike counts generally are close to Poisson (Kluger et al., 2010), notwithstanding the fact that the fine temporal structure is not Poisson. For place cells, Fenton and colleagues (Fenton \& Muller, 1998) find that place cells fire even more variably than would be predicted by a Poisson model; the excess variance is attributable to attention (Fenton et al., 2010) or nonspatial signals that modulate the firing rate but not the location of place cell firing (Leutgeb, Leutgeb, Moser, \& Moser, 2005; Jackson \& Redish, 2007). The spatial resolution of a place code should suffer when the position signal is conflated with other signals, providing one more reason that the grid code in $\mathrm{mEC}$ might be better suited for integrating path information than the place code in CA1. Both place cells and grid cells encode position not only in the firing rate but also in the timing of spikes relative to the ongoing theta oscillation (O'Keefe \& Recce, 1993; Hafting et al., 2008). A temporal phase code at the single cell or population level is potentially more precise in resolving spatial location than counting spikes; decoding such a code, however, was beyond the scope of this study.

Estimating the most likely spatial location relies on having full knowledge of the place and grid field firing rate profiles at each location. For the grid code, the lattices need not be perfectly regular to achieve high spatial resolution. What is required is simply a disjunctive union of intervals at successively finer spatial scales; the periodicity of the intervals is irrelevant. For instance, applying different lateral shifts to different firing fields of within one module would disrupt the periodicity but not change the resolution. Moreover, the existence of modules, defined as subpopulations of neurons whose grid fields have the same spacing, is not truly required. Each grid cell can possess its own lattice spacing, drawn from the entire continuum of possible length scales. As long as all length scales are densely represented, maximum likelihood decoding of the population response will be highly accurate and subject to low error.

On the other hand, both periodicity and modularity are crucial for the modular arithmetic scheme. The spatial range, defined as the maximum distance that is uniquely represented by the set of all modules, is unbounded in the absence of noise, leading to the remarkable property that a huge spatial range, on the order of kilometers, could be supported by modules with $\lambda_{i}$ 's ranging from 30 to 70 centimeters (Fiete et al., 2008). To extend the spatial range beyond the maximum grid period, Fiete et al. proposed that the spatial periods should not be multiples of each other or, more 
generally, have common divisors. Such a constraint can be satisfied aptly by a set of close spatial periods; indeed, the largest spatial range will be obtained when the periods cluster near the maximal period. In the presence of noise, though, narrow spatial periods make the grid code excruciatingly prone to error, leading to a dramatic loss of spatial resolution. In principle, these problems can be overcome by adding redundancy, using modules with very low errors and fine correction algorithms, yet this is a nontrivial challenge. In addition, the grid modules should be highly stable over time for such computations to be feasible. Experimental results indicate that the spatial periods rescale in response to changing the geometry of the environment (Derdikman et al., 2009) or the context (Fyhn, Hafting, Treves, Moser, \& Moser, 2007), and in general exhibit a high variability between trials (Brun et al., 2008; Kluger et al., 2010; Reifenstein et al., 2010). While variability may greatly diminish the effective spatial range of a grid code, the local resolution can still be sufficiently high, as we have shown. In this interpretation, the entorhinal cortex's function is to locally represent the animal's position with high resolution, using grid-based coordinate maps that are continually reset and calibrated by landmarks or spatial memory via the hippocampus (McNaughton et al., 2006).

Grid coding maintains its advantage over place coding even in higherdimensional stimulus spaces. For up to forty grid or place cells encoding a two-dimensional environment, Guanella and Verschure were able to show that the position reconstruction error is smaller for the grid code than the place code, as long as one of the following conditions is met: either both the phases and orientations of the grid must vary, or the phasings and spacings (Guanella \& Verschure, 2007). For a grid cell encoding more than one stimulus dimension, the average Fisher information of the population scales as $\lambda^{-2}$ in each dimension. Indeed, if the tuning curve is separable into its individual components (i.e., dimensions), then the Fisher information of grid cell is simply related to the Fisher information of a place cell with a comparable tuning curve width:

$$
\overline{J_{G C}} \sim\left(\begin{array}{ccc}
\frac{1}{\lambda_{1}} & & 0 \\
& \ddots & \\
0 & & \frac{1}{\lambda_{N}}
\end{array}\right) \cdot \overline{J_{P C}} .
$$

In general, the Fisher information is a matrix, which is diagonal in simple cases. The more general case, for tuning curves that are periodic on arbitrary lattices in more than one dimension, is treated by Mathis, Herz, and Stemmler (2012); Mathis, Stemmler, and Herz (2011).

Given that the grid code can be orders of magnitude better than the place code, based on the mean maximum likelihood error (MMLE), why are both codes used? Hippocampus may have 10 times as many neurons as 
medial entorhinal cortex (Mulders et al., 1997) but achieves the same spatial resolution based on these arguments. Yet grid codes and place codes may well serve different purposes. Entorhinal cortex draws on head-direction and velocity inputs (Sargolini et al., 2006), integrating over the path of motion. Grid lattice representations of the external world are well suited for dead reckoning during navigation. As the hippocampus is essential for forming new episodic memories (O'Keefe \& Nadel, 1978), we speculate that place fields are needed for associating specific events with specific locations. Synaptic plasticity and long-term potentiation occur between pairs of cells, so that if the firing of a single cell already represents a unique location, synapses can easily adapt to the conjunction of location and sensory information. A distributed representation of location, as in a grid code, is less suited for forming such associations.

\section{Appendix: Analytical Derivation and Numerical Methods}

A.1 Fisher Information of Grid and Place Cell. The average Fisher information of a place cell $\overline{J_{P C}}$, was defined in equation 3.13, which stated:

$$
\overline{J_{P C}}=\int_{0}^{1} \int_{0}^{1} f_{\max } T \cdot \frac{(x-c)^{2}}{\sigma^{4}} \exp \left(-\frac{(x-c)^{2}}{2 \sigma^{2}}\right) d x d c
$$

Here the details of the computation are given. The inner integral from equation A.1 can be simplified by applying integration by parts:

$$
\begin{aligned}
\int_{0}^{1} & \frac{(x-c)^{2}}{\sigma^{4}} \exp \left(-\frac{(x-c)^{2}}{2 \sigma^{2}}\right) d x \\
= & {\left[-\frac{x}{\sigma^{2}} \exp \left(-\frac{x^{2}}{2 \sigma^{2}}\right)+\frac{\sqrt{2 \pi}}{\sigma} \cdot \operatorname{erf}\left(\frac{x}{\sqrt{2} \sigma}\right)\right]_{-c}^{1-c}=} \\
= & \frac{c-1}{\sigma^{2}} \exp \left(-\frac{(c-1)^{2}}{2 \sigma^{2}}\right)-\frac{c}{\sigma^{2}} \exp \left(-\frac{c^{2}}{2 \sigma^{2}}\right) \\
& -\frac{\sqrt{2 \pi}}{2 \sigma} \cdot\left(\operatorname{erf}\left(\frac{c-1}{\sqrt{2} \sigma}\right)-\operatorname{erf}\left(\frac{c}{\sqrt{2} \sigma}\right)\right) .
\end{aligned}
$$

In order to obtain equation A.1, one has to integrate the result over $c \in[0,1]$. For the first two terms of equation A.2, since $-\exp \left(-\frac{x^{2}}{2 \sigma^{2}}\right)$ is a primitive for 
$\frac{x}{2 \sigma^{2}} \cdot \exp \left(-\frac{x^{2}}{2 \sigma^{2}}\right)$, we obtain

$$
\left[-\exp \left(-\frac{(c-1)^{2}}{2 \sigma^{2}}\right)+\exp \left(-\frac{c^{2}}{2 \sigma^{2}}\right)\right]_{0}^{1}=2 \cdot\left(\exp \left(-\frac{1}{2 \sigma^{2}}\right)-1\right)
$$

and for the second part again by integration by parts:

$$
\begin{aligned}
- & \frac{\sqrt{2 \pi}}{2 \sigma} \cdot \int_{0}^{1}\left(\operatorname{erf}\left(\frac{c-1}{\sqrt{2} \sigma}\right)-\operatorname{erf}\left(\frac{c}{\sqrt{2} \sigma}\right)\right) d c \\
& =\frac{\sqrt{2 \pi}}{2 \sigma} \cdot \sqrt{2} \sigma \cdot\left(\int_{-\frac{1}{\sqrt{2} \sigma}}^{0}-\operatorname{erf}(s) d s+\int_{0}^{\frac{1}{\sqrt{2} \sigma}} \operatorname{erf}(s) d s\right) \\
& =\sqrt{\pi} \cdot 2 \cdot \int_{0}^{\frac{1}{\sqrt{2} \sigma}} \operatorname{erf}(s) d s \\
& =2 \sqrt{\pi} \cdot\left[s \cdot \operatorname{erf}(s)+\frac{1}{\sqrt{\pi}} \exp \left(-s^{2}\right)\right]_{0}^{\frac{1}{\sqrt{2} \sigma}} \\
& =2 \sqrt{\pi}\left(\frac{1}{\sqrt{2} \sigma} \operatorname{erf}\left(\frac{1}{\sqrt{2} \sigma}\right)+\frac{1}{\sqrt{\pi}} \exp \left(-\frac{1}{2 \sigma^{2}}\right)-\frac{1}{\sqrt{\pi}} \cdot \exp (0)\right) \\
& =\frac{\sqrt{2 \pi}}{\sigma} \operatorname{erf}\left(\frac{1}{\sqrt{2} \sigma}\right)+2 \cdot \exp \left(-\frac{1}{2 \sigma^{2}}\right)-2 .
\end{aligned}
$$

When equations A.3 and A.4 are summed, the average Fisher information of a place cell, equation A.1, is

$$
\overline{J_{P C}}=f_{\max } T \cdot\left(\frac{\sqrt{2 \pi}}{\sigma} \operatorname{erf}\left(\frac{1}{\sqrt{2} \sigma}\right)+4 \cdot \exp \left(-\frac{1}{2 \sigma^{2}}\right)-4\right) .
$$

The second and third terms together behave like a staircase function that is zero for large $\sigma$ and quickly approaches -4 for small values. The first term is the leading term, where $\operatorname{erf}\left(\frac{1}{\sqrt{2} \sigma}\right) \approx 1$ for $\sigma<1$. Hence, the average Fisher information scales as $\propto \frac{1}{\sigma}$ for small $\sigma$. The other terms change the behavior slightly, contributing a bend to the curve for $\sigma>0.1$ in Figure 4a. This result is reported in the main text in equation 3.14.

The average firing rate of a place cell can be calculated as follows:

$$
\overline{f_{P C}}=\int_{0}^{1} \int_{0}^{1} f_{\max } \exp \left(-\frac{(x-c)^{2}}{2 \sigma^{2}}\right) d x d c
$$


Analogous to the average Fisher information, this integral can be computed by

$$
\begin{aligned}
\overline{f_{P C}} & =\sqrt{2} \sigma f_{\max } \int_{0}^{1} \int_{-\frac{c}{\sqrt{2} \sigma}}^{\frac{1-c}{\sqrt{2} \sigma}} \exp \left(-t^{2}\right) d t d c \\
& =\sqrt{2} \sigma f_{\max } \int_{0}^{1} \frac{\sqrt{\bar{\pi}}}{2}\left(-\operatorname{erf}\left(\frac{c-1}{\sqrt{2} \sigma}\right)+\operatorname{erf}\left(\frac{c}{\sqrt{2} \sigma}\right)\right) d c \\
& =f_{\max }\left(\sqrt{2 \pi} \sigma \operatorname{erf}\left(\frac{1}{\sqrt{2} \sigma}\right)+2 \sigma^{2} \cdot \exp \left(-\frac{1}{2 \sigma^{2}}\right)-2 \sigma^{2}\right)
\end{aligned}
$$

where the last equation followed from equation A.4.

Next, we present the computation of the average Fisher information of a grid cells $\overline{J_{G C}}$, as defined in equation 3.16 , which stated:

$$
\overline{J_{G C}}=\frac{1}{\lambda} \cdot \int_{0}^{\lambda} \int_{0}^{1} J_{G C}(x, \varphi) d x d \varphi .
$$

Due to the periodicity of $J_{G C}(x, \varphi)$ in $x, \overline{J_{G C}}$ need be integrated over only half of the periodic domain, followed by multiplication with $2 / \lambda$. Furthermore, for small periods, averaging over different phases is not necessary, again due to the periodicity. Hence, for small $\lambda$, the case we are actually interested in is

$$
\overline{J_{G C}}=\frac{1}{\lambda} \cdot \int_{0}^{\lambda} \int_{0}^{1} J_{G C}(x, \varphi) d x d \varphi \approx \frac{2 f_{\max } T}{\lambda} \int_{0}^{\lambda / 2} \frac{x^{2}}{\sigma^{4}} \exp \left(-\frac{x^{2}}{2 \sigma^{2}}\right) d x .
$$

The last integral can be computed by similar means as above:

$$
\begin{aligned}
& \int_{0}^{\lambda / 2} \frac{-x}{\sigma^{2}} \cdot \underbrace{\frac{-x}{\sigma^{2}} \exp \left(-\frac{x^{2}}{2 \sigma^{2}}\right)}_{=\left(\exp \left(-\frac{x^{2}}{2 \sigma^{2}}\right)\right)^{\prime}} d x \\
& =\left[\frac{-x}{\sigma^{2}} \exp \left(-\frac{x^{2}}{2 \sigma^{2}}\right)\right]_{0}^{\lambda / 2}+\frac{1}{\sigma^{2}} \cdot \int_{0}^{\lambda / 2} \exp \left(-\frac{x^{2}}{2 \sigma^{2}}\right) d x \\
& =\frac{-\lambda}{2 \sigma^{2}} \exp \left(-\frac{\lambda^{2}}{8 \sigma^{2}}\right)+\frac{\sqrt{2}}{\sigma} \cdot \int_{0}^{\frac{\lambda}{2 \sqrt{2} \sigma}} \exp \left(-t^{2}\right) d t \\
& =\frac{-\lambda}{2 \sigma^{2}} \exp \left(-\frac{\lambda^{2}}{8 \sigma^{2}}\right)+\frac{\sqrt{2 \pi}}{2 \sigma} \operatorname{erf}\left(\frac{\lambda}{2 \sqrt{2} \sigma}\right) .
\end{aligned}
$$


Thus, equation A.8 becomes

$$
\overline{J_{G C}}=f_{\max } T\left(\frac{\sqrt{2 \pi}}{\sigma \lambda} \operatorname{erf}\left(\frac{\lambda}{2 \sqrt{2} \sigma}\right)-\frac{1}{\sigma^{2}} \exp \left(-\frac{\lambda^{2}}{8 \sigma^{2}}\right)\right) .
$$

In terms of the area ratio $r_{A}$ from equation 3.18, that is, $\sigma=\frac{r_{A} \cdot \lambda}{2 \sqrt{-2 \log (\beta)}}$, we can write

$$
\begin{aligned}
& \overline{J_{G C}} \\
& =\frac{f_{\max } T}{r_{A} \cdot \lambda^{2}} \underbrace{\left(2 \sqrt{-4 \pi \log (\beta)} \operatorname{erf}\left(\frac{\sqrt{-\log (\beta)}}{r_{A}}\right)-\frac{8 \log (\beta)}{r_{A}} \exp \left(\frac{\log (\beta)}{r_{A}^{2}}\right)\right)}_{=: f\left(r_{A}, \beta\right)} .
\end{aligned}
$$

For the parameters we are interested in, $\beta=0.05$ and $r_{A}<0.5$, the right term is negligible and the first term is effectively constant in for $r_{A}<0.5$. Therefore, $\overline{J_{G C}} \propto \frac{f_{\max }}{r_{A} \cdot \lambda^{2}}$ (see equation 3.17).

Here we derived an approximation for small spatial periods. For larger spatial periods, there will be boundary effects when averaging over the spatial periods. However, numerical comparison showed that the derived formula for $\overline{J_{G C}}$ gives a good approximation, even for spatial periods close to one.

For the grid cell, the average rate is defined by

$$
\overline{f_{G C}}=\frac{1}{\lambda} \int_{0}^{\lambda} \int_{0}^{1} \alpha(x, \varphi) d x d \varphi
$$

with $\alpha$ being the firing rate as defined in equation 3.3, but with spatial phase $\varphi$. Analogous to the average Fisher information, this value can be approximately computed by

$$
\begin{aligned}
\overline{f_{G C}} & \approx \frac{2}{\lambda} \int_{0}^{\frac{\lambda}{2}} f_{\max } \cdot \exp \left(-\frac{x^{2}}{2 \sigma^{2}}\right) d x=\frac{2 \sqrt{2} f_{\max } \sigma}{\lambda} \int_{0}^{\frac{\lambda}{2 \sqrt{2} \sigma}} \cdot \exp \left(-t^{2}\right) d t \\
& =\frac{\sqrt{2 \pi} f_{\max } \sigma}{\lambda} \operatorname{erf}\left(\frac{\lambda}{2 \sqrt{2} \sigma}\right)=f_{\max } \cdot \frac{\sqrt{2 \pi} r_{A}}{2 \sqrt{-2 \log (\beta)}} \operatorname{erf}\left(\frac{\sqrt{-\log (\beta)}}{r_{A}}\right) .
\end{aligned}
$$

Whereas the average firing rate for a place cell is characterized by linear growth in $\sigma$, the average firing rate of a grid cell remains constant for 
changing spatial periods, because the firing field size is determined by the spatial period. This manifests itself in that the average Fisher information per average firing rate falls with the inverse square of $\lambda$ and $\sigma$ for grid and place cells, respectively.

A.2 Monte Carlo Integration and MMLE. The mean maximum likelihood error (MMLE) is best computed by Monte Carlo integration. Each set of parameters governing a grid or place code determines a joint probability distribution $P(K, x)$, from which we realized samples $\left(x_{l}, K_{l}\right)_{1 \leq l \leq R}$ with $R \geq 10^{5}$. From these realizations, we compute

$$
\chi_{M L E}^{2} \approx \frac{1}{R} \sum_{l=1}^{R}\left(x_{l}-\hat{x}_{M L E}\left(K_{l}\right)\right)^{2}=: \widehat{\chi}_{M L E}^{2}(R)
$$

The right-hand side converges with $\frac{1}{\sqrt{R}}$ toward the MMLE. The Monte Carlo integration is stopped if

$$
\left|\widehat{\chi}_{M L E}^{2}(R)-\widehat{\chi}_{M L E}^{2}\left(R+10^{4}\right)\right|<1.001 \cdot \widehat{\chi}_{M L E}^{2}\left(R+10^{4}\right) .
$$

In other words, convergence is said to be reached when the $\widehat{\chi}_{M L E}^{2}$ changes by less than $10^{-3}$ over the last 10000 iterations. A similar convergence criteria was used in Bethge et al. (2002). As an additional test, we corroborate the error estimates by bootstrapping methods.

\section{Acknowledgments}

We thank Carleen Kluger for helpful comments on earlier drafts of the manuscript and Dinu Paterniche for his graphics support. This work was supported by the Federal Ministry for Education and Research (through the Bernstein Center for Computational Neuroscience Munich 01GQ0440).

\section{References}

Attneave, F. (1954). Some informational aspects of visual perception. Psychological Review, 61(3), 183-193.

Barlow, H. (1959). Sensory mechanisms, the reduction of redundancy, and intelligence. In Her Majesty's Stationery Office (Ed.), The mechanisation of thought processes (Vol. 10, pp. 535-539). London: Her Majesty's Stationery Office.

Barry, C., Hayman, R., Burgess, N., \& Jeffery, K. (2007). Experience-dependent rescaling of entorhinal grids. Nature Neuroscience, 10(6), 682-684.

Bethge, M., Rotermund, D., \& Pawelzik, K. (2002). Optimal short-term population coding: When Fisher information fails. Neural Comput., 14, 2317-2351. 
Bobrowski, O., Meir, R., \& Eldar, Y. (2009). Bayesian filtering in spiking neural networks: Noise, adaptation, and multisensory integration. Neural Comput., 21(5), 1277-1320.

Boccara, C., Sargolini, F., Thoresen, V., Solstad, T., Witter, M., Moser, E., et al. (2010). Grid cells in pre- and parasubiculum. Nature Neuroscience, 13(8), 987-994.

Bragin, A., Jando, G., Nadasdy, Z., Hetke, J., Wise, K., \& Buzsaki, G. (1995). Gamma (40-100 Hz) oscillation in the hippocampus of the behaving rat. J. Neuroscience, 15(1), 47-60.

Brown, W., \& Bäcker, A. (2006). Optimal neuronal tuning for finite stimulus spaces. Neural Comput., 18, 1511-1526.

Brun, V., Solstad, T., Kjelstrup, K., Fyhn, M., Witter, M., Moser, E., et al. (2008). Progressive increase in grid scale from dorsal to ventral medial entorhinal cortex. Hippocampus, 18, 1200-1212.

Brunel, N., \& Nadal, J.-P. (1998). Mutual information, Fisher information, and population coding. Neural Comput., 10, 1731-1757.

Burak, Y., Brookings, T., \& Fiete, I. (2006). Triangular lattice neurons may implement an advanced numeral system to precisely encode rat position over large ranges. arXiv:q-bio/0606005v1, 93106:4.

Burak, Y., \& Fiete, I. (2009). Accurate path integration in continuous attractor network models of grid cells. PLoS Computational Biology, 5(2), 1-16.

Burgess, N. (2008). Grid cells and theta as oscillatory interference: Theory and predictions. Hippocampus, 1174, 1157-1174.

Burgess, N., Barry, C., \& O'Keefe, J. (2007). An oscillatory interference model of grid cell firing. Hippocampus, 17, 801-812.

Buzsaki, G. (2006). Rhythms of the brain. New York: Oxford University Press.

Cheng, S., \& Loren, F. (2010). From grid cells to place cells: A generic and robust principle accounts for multiple spatial maps. Frontiers in Computational Neuroscience. Conference Abstract: Bernstein Conference on Computational Neuroscience. doi: 10.3389/conf.fncom.2010.51.00125.

Derdikman, D., Whitlock, J., Tsao, A., Fyhn, M., Hafting, T., Moser, M.-B., et al. (2009). Fragmentation of grid cell maps in a multicompartment environment. Nature Neuroscience, 12(10), 1325-1332.

Deshmukh, S., Yoganarasimha, D., Voicu, H., \& Knierim, J. (2010). Theta modulation in the medial and the lateral entorhinal cortex. J. Neurophysiology, 104, 9941006.

Doeller, C., Barry, C., \& Burgess, N. (2010). Evidence for grid cells in a human memory network. Nature, 463(7281), 657-661.

Eurich, C., \& Wilke, S. (2000). Multidimensional encoding strategy of spiking neurons. Neural Comput., 12(7), 1519-1529.

Fenton, A., Kao, H.-Y., Neymotin, S., Olypher, A., Vayntrub, Y., Lytton, W., et al. (2008). Unmasking the CA1 ensemble code by exposures to small and large environments: More place cells and multiple, irregularly arranged and expanded place fields in the larger space. J. Neuroscience, 28(44), 1125011262.

Fenton, A., Lytton, W., Barry, J., Lenck-Santini, P.-P., Zinyuk, L., Kubík, S., et al. (2010). Attention-like modulation of hippocampus place cell discharge. J. Neuroscience, 30(13), 4613-4625. 
Fenton, A., \& Muller, R. (1998). Place cell discharge is extremely variable during individual passes of the rat through the firing field. Proceedings of the National Academy of Sciences USA, 95, 3182-3187.

Fiete, I., Burak, Y., \& Brookings, T. (2008). What grid cells convey about rat location. J. Neuroscience, 28(27), 6858-6871.

Franzius, M., Vollgraf, R., \& Wiskott, L. (2007). From grids to places. J. Computational Neuroscience, 22, 297-299.

Fuhs, M., \& Touretzky, D. (2006). A spin glass model of path integration in rat medial entorhinal cortex. J. Neuroscience, 26(16), 4266-4276.

Fyhn, M., Hafting, T., Treves, A., Moser, M.-B., \& Moser, E. (2007). Hippocampal remapping and grid realignment in entorhinal cortex. Nature, 446(7132), 190 194.

Fyhn, M., Molden, S., Witter, M., Moser, E., \& Moser, M.-B. (2004). Spatial representation in the entorhinal cortex. Science, 305(5688), 1258-1264.

Garden, D., Dodson, P., O'Donnell, C., White, M., \& Nolan, M. (2008). Tuning of synaptic integration in the medial entorhinal cortex to the organization of grid cell firing fields. Neuron, 60(5), 875-889.

Geisler, C., Diba, K., Pastalkova, E., Mizuseki, K., Royer, S., \& Buzsáki, G. (2010). Temporal delays among place cells determine the frequency of population theta oscillations in the hippocampus. Proceedings of the National Academy of Sciences USA, 107(17), 7957-7962.

Giocomo, L., Zilli, E., Fransén, E., \& Hasselmo, M. (2007). Temporal frequency of subthreshold oscillations scales with entorhinal grid cell field spacing. Science, 315(5819), 1719-1722.

Guanella, A., \& Verschure, P. (2007). Prediction of the position of an animal based on populations of grid and place cells: A comparative study. J. Integrative Neuroscience, 6(3), 433-446.

Hafting, T., Fyhn, M., Bonnevie, T., Moser, M.-B., \& Moser, E. (2008). Hippocampusindependent phase precession in entorhinal grid cells. Nature, 453(7199), 1248 1252.

Hafting, T., Fyhn, M., Molden, S., Moser, M.-B., \& Moser, E. (2005). Microstructure of a spatial map in the entorhinal cortex. Nature, 436(7052), 801-806.

Hasselmo, M., Giocomo, L., \& Zili, E. (2007). Grid cell firing may arise from interference of theta frequency membrane potential oscillations in single neurons. Hippocampus, 17(12), 1252-1271.

Huys, Q., Zemel, R., Natarajan, R., \& Dayan, P. (2007). Fast population coding. Neural Comput., 19(2), 404-441.

Itskov, V., Pastalkova, E., Mizuseki, K., Buzsaki, G., \& Harris, K. (2008). Thetamediated dynamics of spatial information in hippocampus. J. Neuroscience, 28(23), 5959-5964.

Jackson, J., \& Redish, D. (2007). Network dynamics of hippocampal cell-assemblies resemble multiple spatial maps within single tasks. Hippocampus, 17, 1209-1229.

Jeffery, K. (2008). Self-localization and the entorhinal-hippocampal system. Current Opinion in Neurobiology, 17, 1-8.

Kjelstrup, K., Solstad, T., Brun, V., Hafting, T., Leutgeb, S., Witter, M., et al. (2008). Finite scale of spatial representation in the hippocampus. Science, 321(5885), 140143. 
Kluger, C., Mathis, A., Stemmler, M., \& Herz, A. (2010). Movement related statistics of grid cell firing. Frontiers in Computational Neuroscience. Conference Abstract: Bernstein Conference on Computational Neuroscience, 4. doi: 10.3389/conf.fncom.2010 .51 .00134 .

Kropff, E., \& Treves, A. (2008). The emergence of grid cells: Intelligent design or just adaptation? Hippocampus, 18, 1256-1269.

Lehmann, E., \& Casella, G. (1998). Theory of point estimation (2nd ed.). New York: Springer-Verlag.

Leutgeb, S., Leutgeb, J., Moser, M.-B., \& Moser, E. (2005). Place cells, spatial maps and the population code for memory. Current Opinion in Neurobiology, 15(6), 738746.

Leutgeb, J. K., Leutgeb, S., Moser, M.-B., \& Moser, E. I. (2007). Pattern separation in the dentate gyrus and CA3 of the hippocampus. Science, 315(5814), 961-966.

Leutgeb, S., Leutgeb, J., Treves, A., Moser, M.-B., \& Moser, E. (2004). Distinct ensemble codes in hippocampal areas CA3 and CA1. Science, 305(5688), 1295-1298.

Mathis, A., Herz, A.V.M., \& Stemmler, M. (2012). The resolution of nested neuronal representations can be exponential in the number of neurons. Accepted, Physical Review Letters.

Mathis, A., Stemmler, M., \& Herz, A. (2010). How good is grid coding versus place coding for navigation using noisy, spiking neurons? BMC Neuroscience, 11(Suppl. 1), O20.

Mathis, A., Stemmler, M., \& Herz, A. (2011). Exponential scaling of nested neuronal representations. Front. Comput. Neurosci. Conference Abstract: BC11: Computational Neuroscience and Neurotechnology Bernstein Conference \& Neurex Annual Meeting.

McNaughton, B., Battaglia, F., Jensen, O., Moser, E., \& Moser, M.-B. (2006). Path integration and the neural basis of the "cognitive map." Nature Reviews. Neuroscience, 7(8), 663-678.

Mhatre, H., Gorchetchnikov, A., \& Grossberg, S. (2010). Grid cell hexagonal patterns formed by fast self-organized learning within entorhinal cortex. Hippocampus, 22, 320-334.

Mulders, W., West, M., \& Slomianka, L. (1997). Neuron numbers in the presubiculum, parasubiculum, and entorhinal area of the rat. J. Comparative Neurology, 385(1), 83-94.

O'Keefe, J. (1976). Units in the hippocampus moving rat. Experimental Neurology, 51, 78-109.

O'Keefe, J., \& Dostrovsky, J. (1971). The hippocampus as a spatial map: Preliminary evidence from unit activity in the freely-moving rat. Brain Research, 34, 171-175.

O'Keefe, J., \& Nadel, L. (1978). The hippocampus as a cognitive map. New York: Oxford University Press.

O'Keefe, J., \& Recce, M. (1993). Phase relationship between hippocampal place units and the EEG theta rhythm. Hippocampus, 3(3), 317-330.

Paradiso, M. (1988). A theory for the use of visual orientation information which exploits the columnar structure of striate cortex. Biological Cybernetics, 58, 35-49.

Pouget, A., Dayan, P., \& Zemel, R. (2003). Inference and coding with population codes. Annu. Rev. Neurosci., 26, 381-410.

Pouget, A., Deneve, S., Ducom, J., \& Latham, P. (1999). Narrow versus wide tuning curves: What's best for a population code? Neural Comput., 11, 85-90. 
Quilichini, P., Sirota, A., \& Buzsaki, G. (2010). Intrinsic circuit organization and thetagamma oscillation dynamics in the entorhinal cortex of the rat. J. Neuroscience, 30(33), 11128-11142.

Reifenstein, E., Stemmler, M., \& Herz, A. (2010). Single-run phase precession in entorhinal grid cells. Frontiers in Computational Neuroscience. Conference Abstract: Bernstein Conference on Computational Neuroscience. doi:10.3389/conf.fncom.2010 .51 .00093 .

Remme, M., Lengyel, M., \& Gutkin, B. (2010). Democracy-independence trade-off in oscillating dendrites and its implications for grid cells. Neuron, 66(3), 429-437.

Rolls, E., Stringer, S., \& Elliot, T. (2006). Entorhinal cortex grid cells can map to hippocampal place cells by competitive learning. Network: Computation in Neural Systems, 447, 447-465.

Salinas, E., \& Abbott, L. (1994). Vector reconstruction from firing rates. J. Computational Neuroscience, 1, 89-107.

Sargolini, F., Fyhn, M., Hafting, T., McNaughton, B., Witter, M., Moser, M.-B., et al. (2006). Conjunctive representation of position, direction, and velocity in entorhinal cortex. Science, 312(5774), 758-762.

Seung, H., \& Sompolinsky, H. (1993). Simple models for reading neuronal population codes. Proceedings of the National Academy of Sciences USA, 90(22), 10749-10753.

Shadlen, M., \& Newsome, W. (1998). The variable discharge of cortical neurons: Implications for connectivity, computation, and information coding. J. Neuroscience, 18(10), 3870-3896.

Si, B., \& Treves, A. (2009). The role of competitive learning in the generation of DG fields from EC inputs. Cogn. Neurodyn., 3, 177-187.

Softky, W., \& Koch, C. (1993). The highly irregular firing of cortical cells is inconsistent with temporal integration of random EPSPs. J. Neuroscience, 13(1), 334-350.

Solstad, T., Moser, E., \& Einevoll, G. (2006). From grid cells to place cells: A mathematical model. Hippocampus, 16, 1026-1031.

Taube, J., Muller, R., \& Ranck, J. (1990a). Head-direction cells recorded from the postsubiculum in freely moving rats. I. Description and quantitative analysis. $J$. Neuroscience, 10(2), 420-435.

Taube, J., Muller, R., \& Ranck, J. (1990b). Head-direction cells recorded from the postsubiculum in freely moving rats. II. Effects of environmental manipulations. J. Neuroscience, 10(2), 436-447.

Wilke, S., \& Eurich, C. (2002). Representational accuracy of stochastic neural populations. Neural Comput., 14, 155-189.

Wilson, M., \& McNaughton, B. (1993). Dynamics of the hippocampal ensemble code for space. Science, 261(5124), 1055-1058.

Zhang, K., \& Sejnowski, T. (1999). Neuronal tuning: To sharpen or broaden? Neural Comput., 11(1), 75-84.

Zilli, E., \& Hasselmo, M. (2010). Coupled noisy spiking neurons as velocitycontrolled oscillators in a model of grid cell spatial firing. J. Neuroscience, 30(41), 13850-13860.

Received December 22, 2010; accepted February 7, 2012. 